\title{
An analytical solution to assess the $S H$ seismoelectric response of the vadose zone
}

\author{
L. B. Monachesi, ${ }^{1,2}$ F. I. Zyserman ${ }^{2,3}$ and L. Jouniaux ${ }^{4}$ \\ ${ }^{1}$ Instituto de Investigación en Paleobiología y Geología, Universidad Nacional de Río Negro Av. Roca 1242, Gral. Roca, Río Negro, Argentina. E-mail: \\ ${ }^{2}$ CONICET, Av. Rivadavia 1917, Ciudad Autónoma de Buenos Aires, Argentina \\ ${ }^{3}$ Facultad de Ciencias Astronómicas y Geofisicas, Universidad Nacional de La Plata Paseo del Bosque s/n, B1900FWA La Plata, Argentina \\ ${ }^{4}$ Institut de Physique du Globe de Strasbourg UMR7516, Université de Strasbourg, CNRS, 5 rue René Descartes, 67084 Strasbourg, France
}

Accepted 2018 March 15. Received 2018 March 13; in original form 2017 July 22

\begin{abstract}
SUMMAR Y
We derive an analytical solution of the seismoelectric conversions generated in the vadose zone, when this region is crossed by a pure shear horizontal $(\mathrm{SH})$ wave. Seismoelectric conversions are induced by electrokinetic effects linked to relative motions between fluid and porous media. The considered model assumes a 1D soil constituted by a single layer on top of a half space in contact at the water table, and a shearing force located at the earth's surface as the wave source. The water table is an interface expected to induce a seismoelectric interfacial response (IR). The top layer represents a porous rock in which porous space is partially saturated by water and air, while the half-space is completely saturated with water, representing the saturated zone. The analytical expressions for the coseismic fields and the interface responses, both electric and magnetic, are derived by solving Pride's equations with proper boundary conditions. An approximate analytical expression of the solution is also obtained, which is very simple and applicable in a fairly broad set of situations. Hypothetical scenarios are proposed to study and analyse the dependence of the electromagnetic fields on various parameters of the medium. An analysis of the approximate solution is also made together with a comparison to the exact solution. The main result of the present analysis is that the amplitude of the interface response generated at the water table is found to be proportional to the jump in the electric current density, which in turn depends on the saturation contrast, poro-mechanical and electrical properties of the medium and on the amplitude of the solid displacement produced by the source. This result is in agreement with the one numerically obtained by the authors, which has been published in a recent work. We also predict the existence of an interface response located at the surface, and that the electric interface response is several orders of magnitude bigger than the electric coseismic field, whereas it is the opposite using compressional waves as shown by theoretical and experimental results. This fact should encourage the performance of field and laboratory tests to check the viability of SHTE seismoelectrics as a near surface prospecting/monitoring tool.
\end{abstract}

Key words: Electrical properties; Hydrogeophysics; Permeability and porosity; Wave propagation.

\section{INTRODUCTION}

The seminal work of Pride (1994) completed previous efforts (Frenkel 1944) to build a solid theory to explain both the already observed conversions of seismic waves travelling in the shallow subsurface of the Earth into electromagnetic energy (Ivanov 1939; Long \& Rivers 1975; Thompson \& Gist 1993) and also the conversion of electromagnetic signals into travelling seismic waves. It encouraged several authors to make further studies, trying to appropriately characterize the different signals predicted theoretically and detected both in laboratories and in the field. Among them, we can mention the work of Pride \& Haartsen (1996), who derived Green's functions for electroseismics/seismoelectrics 
for solid and fluid displacements and the electric field; this work was extended by Gao \& Hu (2010), who developed the Green's function for the magnetic field and considered moment tensors as sources. Haartsen \& Pride (1997) used a global matrix method to study the so called interface response (IR) generated at interfaces of a layered Earth illuminated by electromagnetic and seismic point sources. They showed that the electromagnetic IR generated by an impinging compressional seismic wave on the interface between two layers has a pattern which in the near field can be associated with the one of a vertical electric dipole located at the interface, right beneath the seismic source. Again, Hu \& Gao (2011) extended this algorithm and applied it to analyse the electromagnetic fields produced by a finite fault rupture. They observed two types of electric fields: the coseismic electric field (E-Cos), that is, the electromagnetic signal supported within the seismic wave, travelling at its speed and the post-seismic decaying variation. Garambois \& Dietrich (2001), in a work where seismoelectric field signals were analysed, developed transfer functions showing that in the lower frequency band the E-Cos is proportional to the grain acceleration. They also showed that the particle velocity in a seismic shear wave and the magnetic field are related in the same way. Similarly, Pride \& Garambois (2005) proved with numerical studies that compressional or shear waves crossing an interface in which any of the transport properties or elastic moduli change, give rise to electromagnetic disturbances that could be measured at the surface. Moreover, they observed that the amplitude of the converted electric field at the interface can be drastically increased if there is a thin layer of third material present at the interface, and suggested that this feature could be exploited in hydrological applications. We mention here that in Pride's theory, and therefore in all the works previously mentioned, the amplitudes of the coseismic electromagnetic signals are controlled by the properties of the porous material (the formation factor) and by the properties of the pore fluid/solid interface (the zeta potential). In the alternative seismoelectric theory proposed by Revil et al. (2003, 2007), the role of the latter is played by the excess charge per unit pore volume.

Seismoelectrics and electroseismics, which aim at combining the sensitivity of the electric methods to the fluid content with the spatial resolution of the seismic method, have been used to investigate hydrogeological reservoirs (Dupuis et al. 2007; Dupuis et al. 2009; Schakel et al. 2012; Warden et al. 2013), especially in arid environments (Valuri et al. 2012), and hydrocarbon reservoirs (Thompson et al. 2005; Hornbostel \& Thompson 2007; Hu et al. 2007; Thompson et al. 2007; Zyserman et al. 2012; Guan et al. 2013; Zyserman et al. 2015). It has been shown that seismic compressional waves generate IRs with amplitudes smaller than those of the coseismic signals, which makes the former difficult to be measured. As detailed in Jouniaux \& Zyserman (2016), several authors have studied how to deal with this problem. For example, Dupuis et al. (2007) proposed to measure the electric field within a borehole, below the interface of interest and Haines et al. (2007) suggested to implement off-line geometry surveys as a means of separating the IR signal from the coseismic and source related ones. Quite recently, Peng et al. (2017) performed a laboratory study of IRs produced by sandstone targets with different geometries, aiming at characterizing the amplitudes of the IRs produced by incident waves and their reflections at multiple interfaces, and to study the polarity reversals for different setups.

Focusing on the signals processing, Kepic \& Rosid (2004) proposed to create virtual 'super gathers' as a way to obtain a dense spatial sampling, necessary to apply Radon transform filters, also called $(\tau-p)$ where $\tau$ is the intercept time of an event and $p$ the apparent slowness, or frequency-wavenumber $(f-k)$ filters, which can be used to isolate the IRs as Strahser et al. (2007) have shown. More recently, Warden et al. (2012), aiming at better preserving the amplitude of the IRs, developed a new filtering strategy based on the fast discrete curvelet transform, getting better results than when using the Radon transform or $(f-k)$ filtering.

Most of the mentioned works were developed considering a single saturating fluid at full saturation. The effect of moisture on seismoelectrics was studied by Parkhomenko \& Tsze-San (1964), Parkhomenko \& Gaskarov (1971), Gaskarov \& Parkhomenko (1974) and later by Ageeva et al. (1999), at various frequencies. However, it was not possible to deduce any transfer function as a function of water saturation. It was only in the few last years that the attempts to introduce a quantitative effect of the partial saturation in seismoelectrics/electroseismics have been undertaken (Warden et al. 2013; Bordes et al. 2015; Jardani \& Revil 2015; Zyserman et al. 2015). Bordes et al. (2015) extended the original dynamic transfer function between the coseismic seismoelectric signal and the acceleration based on the works from Pride (1994) and Pride \& Haartsen (1996) to partially saturated states. The transfer function was measured in sand during imbibition and drainage experiments by Holzhauer et al. (2016). These authors showed that the behaviour of this transfer function is complex: it is not monotonous with the water saturation, and a switch in polarity was observed at a critical saturation. This behaviour was modelled by taking into account an electrokinetic coupling dependent on the water saturation showing a non-monotonous behaviour, and using an effective fluid modulus obtained from a power law of the saturation or based on the Reuss average.

Turning now the reader's attention to the choice of the seismic source, on the one hand, we have proved in a previous work (Zyserman et al. 2017a) that the signal-to-noise ratio can be higher when using a shear wave source than when using a compressional source, and on the other hand, although up to now shear wave sources have not been tested in seismoelectric field studies, they have been employed in several works aiming at characterizing the shallow subsurface. Konstantaki et al. (2013) performed a detailed study of how a landfill can be monitored via seismic interferometry, using $S H$-wave sources and receivers. Later, Konstantaki et al. (2015) characterized an heterogeneous landfill using seismic and electric resistivity data. For the seismic analysis they used $S$ waves, generated by a horizontal vibrator with a frequency range of 20-300 Hz. We can also mention the work by Krawczyk et al. (2012), who investigated the roof region of a salt diapir in Hamburg, employing an horizontal vibrator as a source, and recorded the seismic responses with SH-geophones; in Krawczyk et al. (2013) the authors describe several case studies of urban geophysics employing the same source-receiver system as in the previous mentioned work and in Pugin et al. (2013) two examples of multicomponent shallow $S$-wave reflection profiling were presented. Quite recently, Beilecke et al. (2016) carried out high resolution, near surface, shear wave reflection seismic measurements to investigate the fault structure in a $\mathrm{CO}_{2}$ deposition site in Australia. We mention, finally, the works of Stucchi et al. (2017) and Comina et al. (2017), integrating $S H$-wave measurements with $P$ 
wave and Love-wave, respectively, to investigate different geological systems to assess landslide risks. The mentioned works show that there is evidence of successful usage of $\mathrm{SH}$ waves to investigate the subsoil.

We analytically show in this work - confirming numerical results mentioned above - that the use of SHTE seismoelectrics could overcome also the difficulties in measuring the IRs, because the electric coseismic signal has a remarkably smaller amplitude than that of the IR. We recall here that SHTE stands for the coupling between shear horizontal seismic waves and transverse electric fields; the other mode is the so called PSVTM, denoting the coupling of $P$ - and $S V$ - seismic waves with the transverse magnetic mode (Haartsen \& Pride 1997; Zyserman et al. 2010, 2012).

Recently Zyserman et al. (2017a) analysed the seismoelectric conversions generated in the vadose zone when this region is crossed by a pure $S H$ wave, instead of a pure compressional $P$-wave as usually studied. With this choice better spatial resolution can be achieved, due to smaller wavelengths (Krawczyk et al. 2012; Konstantaki et al. 2013, 2015). Moreover, the numerical results showed that the seismoelectric conversions induced by a shear wave source yield an electric interfacial response of about three orders of magnitude larger than the coseismic signal amplitude, leading to a possible detection of the water table by seismoelectromagnetic measurements. In the present work, these seismoelectromagnetic conversions induced in the vadose zone are analytically derived for a simple 1D model involving a step-shaped saturation profile. We are able to identify, for both electric and magnetic fields, IRs at the surface and at the water table and also the coseismic signals above and below the water table. Moreover, we can identify how these signals depend on the soil properties in a clear and simple way. We start our work describing the rock physics models we employ for the effective soil properties, afterwards we introduce the 1D SHTE seismoelectric equations and we continue presenting the analytical solution. Then, we present an approximation to the obtained analytical solution, which is quite simple and applicable in a fairly broad set of situations, making it quite useful as an interpretation tool and to test numerical codes. Then, hypothetical examples are proposed in order to analyse the obtained expressions and a parametric study is performed to study the dependence of the electromagnetic fields on various physical parameters of the media. Finally, an analysis of the approximate solution is made, together with a comparison to the exact solution.

\section{THEORETICAL BACKGROUND}

\subsection{Effective model parameters}

We describe now the rock physics models employed to get the different necessary soil parameters. We consider three different materials building the solid matrix, namely sand, silt and clay, and we call $f_{\text {sand }}, f_{\text {silt }}$ and $f_{\text {clay }}$ their respective volume fractions; $f_{\text {sand }}+f_{\text {silt }}+f_{\text {clay }}=1$. The mass density of the aggregate, $\rho_{s}$, is given by the volume weighted mean of the respective components mass densities:

$\rho_{s}=f_{\text {sand }} \rho_{s, \text { sand }}+f_{\text {silt }} \rho_{s, \text { silt }}+f_{\text {clay }} \rho_{s, \text { clay }}$.

For the effective shear and bulk moduli of the aggregate $G_{s}$ and $K_{s}$, respectively, we use the Reuss lower bound (Mavko et al. 2009), calculated in terms of the components volume fractions, shear moduli and bulk moduli of the solid grains as:

$G_{s}=\left(\frac{f_{\text {sand }}}{G_{s, \text { sand }}}+\frac{f_{\text {silt }}}{G_{s, \text { silt }}}+\frac{f_{\text {clay }}}{G_{s, \text { clay }}}\right)^{-1}, \quad K_{s}=\left(\frac{f_{\text {sand }}}{K_{s, \text { sand }}}+\frac{f_{\text {silt }}}{K_{s, \text { silt }}}+\frac{f_{\text {clay }}}{K_{s, \text { clay }}}\right)^{-1}$.

We use Walton model (Mavko et al. 2009) to estimate the solid matrix shear modulus $G$; this choice is appropriate when unconsolidated soils are considered (Pride 2005; Bordes et al. 2015; Dupuy et al. 2016). It reads

$G=\frac{1}{10}\left[\frac{3(1-\phi)^{2} \hat{C}^{2} P}{\pi B^{2}}\right]$, with $B=\frac{1}{4 \pi}\left(\frac{1}{G_{s}}+\frac{1}{G_{s}+\lambda_{c}}\right)$.

In this equation, $\varphi$ is the porosity, $\hat{C}$ is the so called coordination number, and is related to the packing of the spheres building the solid aggregate, $P$ is the hydrostatic pressure and $\lambda_{c}$ is Lamé's coefficient of the effective grain material calculated as $\lambda_{c}=K_{s}-\frac{2}{3} G_{s}$. In this work we consider $\hat{C}=9$ and $P=101325 \mathrm{~Pa}$.

To deal with both saturated and partially saturated porous media within Pride's formulation for seismoelectrics, it is necessary to introduce appropriate effective properties. We perform here this task by following the same approach taken by Zyserman et al. (2012), Smeulders et al. (2013), Warden et al. (2013), Bordes et al. (2015) and Zyserman et al. (2015). For the effective fluid mass density, we use

$\rho_{f}=\rho_{\mathrm{w}} S_{\mathrm{w}}+\rho_{\mathrm{a}}\left(1-S_{\mathrm{w}}\right)$,

where $\rho_{\mathrm{w}}$ and $\rho_{\mathrm{a}}$ are the densities and $S_{\mathrm{w}}$ and $S_{\mathrm{a}}=1-S_{\mathrm{w}}$ are the saturation degrees of water and air, respectively. The bulk density $\rho_{\mathrm{b}}$ is computed as:

$\rho_{\mathrm{b}}=\phi \rho_{f}+(1-\phi) \rho_{s}$.

The effective viscosity is computed, following Teja \& Rice (1981), in terms of the water viscosity $\beta_{\mathrm{w}}$, air viscosity $\beta_{\mathrm{a}}$ and water saturation $S_{\mathrm{w}}$ using

$\eta=\eta_{\mathrm{a}}\left(\frac{\eta_{\mathrm{w}}}{\eta_{\mathrm{a}}}\right)^{S_{\mathrm{w}}}$ 
Because in Biot's theory, shear stresses are decoupled from pore pressure and, hence, the shear modulus does not depend on the conditions of drainage, the shear modulus of the saturated rock matrix is assumed to be equal to $G$, the shear modulus of the dry matrix (Kümpel 1991).

On the other hand, we compute the absolute permeability as (Bear 1988):

$\kappa=\frac{\eta_{\mathrm{w}} K}{\rho_{f} g}$,

where $K$ is the hydraulic conductivity of the soil, and $g=9.81 \mathrm{~m} \mathrm{~s}^{-2}$ is the gravity acceleration.

In order to characterize the electrical conductivity of the effective fluid saturated solid matrix, we use the expression recently proposed by Warden et al. (2013), extending Pride's original formula (Pride 1994, eq. 242) to the realm of partially saturated media:

$\sigma\left(S_{\mathrm{w}}, \omega\right)=\frac{S_{\mathrm{w}}^{\hat{n}}}{F} \sigma_{\mathrm{w}}+\frac{2}{F} \frac{C_{\mathrm{em}}+C_{\mathrm{os}}(\omega)}{\Lambda}$.

The first term in this equation is Archie's law for a partially saturated medium, where $F=\phi^{-\hat{m}}$ stands for the formation factor, $\hat{m}$ being the cementation exponent and $\hat{n}$ the Archie's saturation exponent. The electrical conductivity of water containing sodium chloride can be computed as $\sigma_{\mathrm{w}}=\sum_{l=\mathrm{Na}^{+}, \mathrm{Cl}^{-}}\left(e z_{l}\right)^{2} b_{l} N_{l}$, where $e=1.6 \times 10^{-19} \mathrm{C}$ is the electron electric charge, and $z_{l}$ is the ions' valence, taken to be one for both species. The ions' mobility $b_{l}$ and concentration $N_{l}$ (depending on the salinity $C_{0}$ ) are calculated following Carcione et al. (2003). The second term in eq. (8) accounts for the surface conductivity; within this expression, as Pride (1994) stated, the factor $C_{\text {em }}$ is the excess conductance associated with the electromigration of double layer ions; $C_{\mathrm{os}}(\omega)$ is the frequency-dependent electro-osmotic conductance due to electrically induced streaming of the excess double-layer ions. Finally, $\Lambda$ is a fundamental porous material parameter related to the volume-to-surface ratio, and can be obtained from the condition $(\xi \alpha \kappa) /\left(\varphi \Lambda^{2}\right)=1$ (Johnson et al. 1987; Pride 1994); the pore-geometry dependent factor $\xi$ lies in the range $4 \leq \xi \leq 8$ (Pride 1994) and in this work we consider $\xi=8$, which corresponds to a network of variable-radii tubes; $\alpha=\varphi F$ is the tortuosity. We remark here that, as in Brovelli et al. (2005) and Warden et al. (2013), the surface conductivity is assumed to be independent of water saturation, because under realistic saturation ranges (residual water saturation $S_{\mathrm{wr}} \geq 0.1$ ) the thickness of the wetting phase layer on the pore surface is always larger than the Debye length. This also means that all fluid-related properties involved in the calculation of the surface conductivity and of the electrokinetic coupling - see below - are just those of water. Note that another approach, with a water saturation-dependent surface conductivity can also be considered; however, it can be observed that it does not affect significantly the seismoelectromagnetic conversions for the considered soils (Zyserman et al. 2017b).

We propose for the effective fluid saturated media the following electrokinetic coupling, again following Warden et al. (2013) and Bordes et al. (2015):

$L_{0}\left(S_{\mathrm{w}}\right)=-\frac{\phi}{\alpha_{\infty}} \frac{\epsilon_{\mathrm{w}} \zeta}{\eta_{\mathrm{w}}}\left(1-2 \frac{d}{\Lambda}\right) S_{\mathrm{w}}^{\hat{n}} \mathcal{C}\left(S_{\mathrm{w}}\right)$

In this equation $\zeta$ is the zeta potential, $\varepsilon_{\mathrm{w}}$ is the permittivity of water, $d$ is the Debye length and $\mathcal{C}\left(S_{\mathrm{w}}\right)$ is a function relating the streaming potential coefficient obtained under partial saturation conditions to the one corresponding to full saturation conditions. This streaming potential coefficient corresponds to the electric potential difference over the pressure difference at the origin of this electrical signal. If the fluid flow does not induce an electric signal, the streaming potential coefficient is zero, and then the electrokinetic coupling $L_{0}$ is also zero, and no seismo-electromagnetic conversions are possible. For a tutorial on these effects see Jouniaux \& Ishido (2012). In eq. (9), we use the following relation, which was experimentally derived from laboratory measurements of the streaming potential coefficient in partially saturated sands and displays a non-monotonic dependence with water saturation (Allègre et al. 2010, 2012, 2014, 2015; Fiorentino et al. 2017):

$\mathcal{C}\left(S_{\mathrm{w}}\right)=\left(\frac{S_{\mathrm{w}}-S_{\mathrm{wr}}}{1-S_{\mathrm{wr}}}\right)\left[1+32\left[1-\left(\frac{S_{\mathrm{w}}-S_{\mathrm{wr}}}{1-S_{\mathrm{wr}}}\right)\right]^{0.4}\right], \quad S_{\mathrm{wr}}=0.20$.

For water saturations below $S_{\mathrm{wr}}, \mathcal{C}\left(S_{\mathrm{w}}\right)$ is set to zero. Others different available models for $\mathcal{C}\left(S_{\mathrm{w}}\right)$ have already been discussed by ourselves and other authors (Strahser et al. 2011) as well, see Zyserman et al. (2017a), Perrier \& Morat (2000), Allègre et al. (2010). We compute the zeta potential as $\zeta=0.008+0.026 \log _{10}\left(C_{0}\right)$ (Pride \& Morgan 1991), and consider a fixed salt concentration $C_{0}=5 \times 10^{-3}$ mol $\mathrm{L}^{-1}$ (Table 1), such that $\zeta=-52 \mathrm{mV}$ throughout this work.

\subsection{SHTE seismoelectric equations}

We model the electromagnetic response to seismic waves generated by a shear source using the equations derived by Pride (1994). In particular, we assume that the source of the system is a shearing force located at the Earth's surface, parallel to the $x$-axis acting on a horizontal infinite plane (see Fig. 1). Under these assumptions both the solid and fluid phases can only undergo displacements in the $x$-direction, with amplitudes depending only on depth $z$; no compressional waves can arise in this model and spherical spreading and Fresnel zone effects are not accounted for. If we also consider that the electroosmotic feedback can be neglected in Biot's equations, as it is usually assumed for frequencies in the range of interest for shallow seismoelectric surveys (10 Hz to $1 \mathrm{kHz}$ ) (Hu \& Liu 2002; Haines \& Pride 2006), Pride's equations can be written in the space-frequency domain, assuming an $e^{i \omega t}$ time dependence, as follows:

$$
-\sigma E_{x}-\frac{\partial H_{y}}{\partial z}=i \omega \frac{\eta}{\kappa} L_{0} u_{f, x}=j_{v},
$$


Table 1. Values of model parameters used in the present study. Those not shown in this table, e.g. effective fluid viscosity $\beta$, effective bulk conductivity $\sigma$, inertial coupling coefficient $g_{0}$, can be obtained from the present ones using the formulae described in the 'Theoretical background' section. Values marked with $\mathrm{a}^{\star}$ symbol correspond to a silt loam; the constituents fractions are chosen according to the USDA soil texture classification triangle (U.S. Soil Conservation Service 1987), the hydraulic parameters are given by Carsel \& Parrish (1998). The vacuum permittivity is taken to be $\varepsilon_{0}=8.85 \times 10^{-12} \mathrm{C}^{2} \mathrm{~N}^{-1} \mathrm{~m}^{-2}$.

\begin{tabular}{llll}
\hline & & Model parameters & \\
\hline$\hat{m}$ & 1.35 & $\rho_{s, \text { sand }}\left(\mathrm{kg} \mathrm{m}^{-3}\right)$ & 2600 \\
$\hat{n}$ & 1.85 & $\rho_{s, \text { silt }}\left(\mathrm{kg} \mathrm{m}^{-3}\right)$ & 2600 \\
$\phi$ & $0.45^{\star}$ & $\rho_{s, \text { clay }}\left(\mathrm{kg} \mathrm{m}^{-3}\right)$ & $1 \times 10^{-3}$ \\
$f_{\text {sand }}$ & $0.3^{\star}$ & $\beta_{w}(\mathrm{~Pa} \mathrm{~s})$ & 1027 \\
$f_{\text {silt }}$ & $0.55^{\star}$ & $\rho_{w}\left(\mathrm{~kg} \mathrm{~m}^{-3}\right)$ & $1.81 \times 10^{-5}$ \\
$f_{\text {clay }}$ & $0.15^{\star}$ & $\beta_{a}\left(\mathrm{~Pa} \mathrm{~s}^{-5}\right.$ & $80 \varepsilon_{0}$ \\
$K\left(\mathrm{~cm} \mathrm{day}{ }^{-1}\right)$ & $10.8^{\star}$ & $\rho_{a}\left(\mathrm{~kg} \mathrm{~m}^{-3}\right)$ & $5 \times 10^{-3}$ \\
$G_{\text {sand }}(\mathrm{GPa})$ & 45 & $\varepsilon_{w}\left(\mathrm{~F} \mathrm{~m}^{-1}\right)$ & 298 \\
$G_{\text {silt }}(\mathrm{GPa})$ & 45 & $C_{0}\left(\mathrm{~mol} \mathrm{~L}^{-1}\right)$ & \\
$G_{\text {clay }}(\mathrm{GPa})$ & 6.8 & $T(\mathrm{~K})$ & \\
$K_{\text {sand }}(\mathrm{GPa})$ & 36 & & \\
$K_{\text {silt }}(\mathrm{GPa})$ & 36 & & \\
$K_{\text {clay }}(\mathrm{GPa})$ & 20.9 & & \\
\hline
\end{tabular}

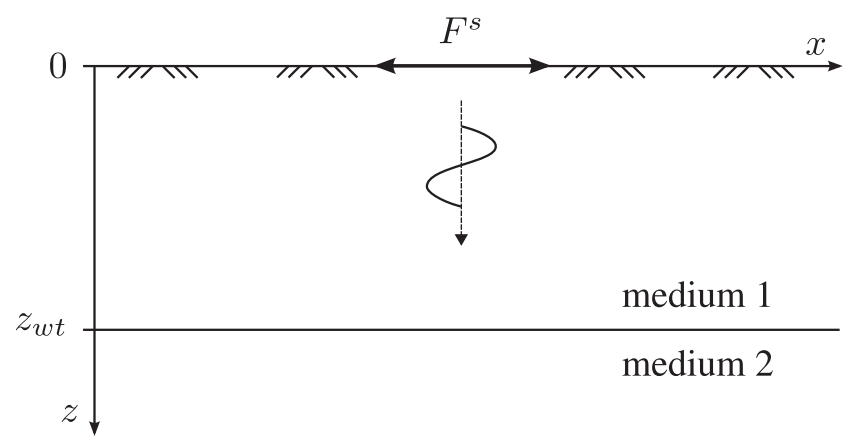

Figure 1. Schematic representation of the seismic shear plane wave travelling downwards in a two-layer 1D system. Medium 1 and medium 2 represent the vadose zone and the saturated zone, respectively, in contact at $z=z_{\mathrm{Wt}}$ (the depth of the water table).

$$
\begin{aligned}
& \frac{\partial E_{x}}{\partial z}+i \omega \mu_{0} H_{y}=0, \\
& -\omega^{2} \rho_{b} u_{s, x}-\omega^{2} \rho_{f} u_{f, x}-G \frac{\partial^{2} u_{s, x}}{\partial z^{2}}=F^{s} \delta(z), \\
& -\omega^{2} \rho_{f} u_{s, x}-\omega^{2} g_{0} u_{f, x}+i \omega \frac{\eta}{\kappa} u_{f, x}=0 .
\end{aligned}
$$

In this system of equations, eqs (11) and (12) are Ampère-Maxwell's equation and Faraday's equation, respectively, - notice that the source of the electromagnetic fields arises due to the electrokinetic coupling -, while eqs (13) and (14) are Biot's equations, being the right-hand side of eq. (13) the seismic source. $E_{x}$ and $H_{y}$ are the electric and magnetic fields, respectively, and $u_{s, x}$ and $u_{f, x}$ are the average solid and relative fluid displacements, respectively. From now on, for the sake of simplicity, we denote $u_{s} \equiv u_{s, x}, u_{f} \equiv u_{f, x}, E \equiv E_{x}$ and $H \equiv H_{y}$. The parameters in eqs (11)-(14) which remain to be mentioned are the vacuum magnetic permeability $\mu_{0}=4 \pi \times 10^{-7} \mathrm{~N} \mathrm{~A}^{-2}$ and the Biot's low-frequency inertial coupling coefficient $g_{0}=\alpha \rho_{f} / \varphi=F \rho_{f}$ (Biot 1956; Santos et al. 2004, 2005; Zyserman et al. 2012). The right-hand side in eq. (11) is the electric current density, source of the electromagnetic signals, and can be referred to as the viscous current density $j_{v}$, whereas $\sigma E$ is the conduction current. The right-hand side of eq. (13) is the shearing source acting on the surface ( $F^{s}$ is the shearing force per unit area).

\section{DERIVATION OF THE ANALYTICAL SOLUTION}

We describe here the geometry of the model, and derive the electric and magnetic fields induced by the shear wave propagation. Our analytical development of the fields allows to clearly individualize both the coseismic fields and the interfacial signals.

\subsection{Electric current density}

Let us consider a porous medium constituted by two regions, schematically depicted in Fig. 1 as a cut in the $x-z$ plane, being $z$ the vertical axis (positive downwards) and $x$ the horizontal axis. The origin of the $z$ coordinates is located at the surface, and both regions are in contact at $z=z_{\mathrm{wt}}$, the depth of the water table. The upper region $\left(0<z<z_{\mathrm{wt}}\right)$ labelled as medium 1 , represents a partially saturated porous rock. This 
space mimics the so called vadose zone where we have added the assumption that the water saturation remains constant with depth. The lower region $\left(z>z_{\mathrm{wt}}\right.$ ), labelled as medium 2, is completely saturated by water, and represents the saturated zone below the water table. Although the proposed model for water saturation distribution is not completely realistic, it is a simple way to describe the water content distribution near the water table, and has allowed us to derive an analytical solution to quantify the effect of the vadose zone on the electromagnetic response, as we describe below.

Biot's eqs (13) and (14) predict that the perturbation created by the shear source at $z=0$ will propagate as a plane wave travelling downwards from the surface to the water table, as is schematically depicted in Fig. 1. Assuming the wave is harmonic with angular frequency $\omega$ and polarized in the $x$-axis direction, the solid and relative fluid displacements in medium $1, u_{s, 1}$ and $u_{f, 1}$ will be given by:

$u_{s, 1}=U_{s, 1} e^{i\left(\lambda_{1} z+\omega t\right)}$ and $u_{f, 1}=U_{f, 1} e^{i\left(\lambda_{1} z+\omega t\right)}$,

where $\lambda_{1}$ is the wavenumber of medium 1 :

$\lambda_{1}=\omega\left[\frac{1}{G}\left(\rho_{b, 1}-\frac{\rho_{f, 1}^{2}}{g_{0,1}-i \eta_{1} /\left(\kappa_{1} \omega\right)}\right)\right]^{1 / 2}$,

being $U_{s, 1}$ and $U_{f, 1}$ the solid and relative fluid displacements at $z=0$. From eq. (16) the $S$-wave velocity $v_{1}$ can be computed as $v_{1}=\omega /\left|\operatorname{Re}\left(\lambda_{1}\right)\right|$. We choose $\lambda_{1}$ such that $\operatorname{Re}\left(\lambda_{1}\right)<0$ in order to consider waves travelling downwards. The two last expressions are obtained by solving eqs (13) and (14) (see Appendix A). Following the same reasoning the solid and relative fluid displacements of medium 2 can be expressed as:

$u_{s, 2}=U_{s, 2} e^{i\left(\lambda_{2}\left(z-z_{w t}\right)+\omega t\right)}$ and $u_{f, 2}=U_{f, 2} e^{i\left(\lambda_{2}\left(z-z_{w t}\right)+\omega t\right)}$,

being $\lambda_{2}$ the wavenumber of medium 2 and $U_{s, 2}$ and $U_{f, 2}$ the corresponding displacements at the water table $\left(\operatorname{Re}\left(\lambda_{2}\right)<0\right)$. Using these expressions in eq. (14), the following relations can be obtained:

$u_{f, 1}=-\frac{\rho_{f, 1}}{g_{0,1}-\frac{i \eta_{1}}{\omega \kappa_{1}}} u_{s, 1}$ and $u_{f, 2}=-\frac{\rho_{f, 2}}{g_{0,2}-\frac{i \eta_{2}}{\omega \kappa_{2}}} u_{s, 2}$.

If an $S H$ wave impinges the interface, there will not occur mode conversions to $P$ or $S V$ waves. So, there will be only three waves to consider: incident $S H$, reflected $S H$ and transmitted $S H$. Now, let us assume that the solid matrix of the porous medium is the same for both media (they only differ in fluid content). In this scenario, the shear modulus is the same for both media, and the reflection coefficient for the relative solid displacement in the case of normal incidence can be computed after Liu \& Greenhalgh (2014) as follows:

$R_{S H}=\frac{\lambda_{1}-\lambda_{2}}{\lambda_{1}+\lambda_{2}}$

Given that $\lambda_{1} \simeq \lambda_{2}$, then $R_{S H} \simeq 0$, and there is no reflection from the interface. Then, the interface is virtually transparent, the wave will pass through it unaffected and the solid displacement at $z=z_{\mathrm{wt}}$ will be the same for both incident and transmitted waves. This allows to write $u_{s, 1}\left(z_{\mathrm{wt}}\right)=u_{s, 2}\left(z_{\mathrm{wt}}\right)$, which is equivalent to write $U_{s, 1} e^{i \lambda_{1} z_{\mathrm{wt}}}=U_{s, 2}$. Moreover, being the solid matrix the same for both regions, the hydraulic permeability will also be the same, so we can write $\kappa_{1}=\kappa_{2}=\kappa$.

The viscous current density $j_{v}$ generated by the charges dragged by the relative fluid motion can be obtained for medium 1 as follows (see eq. 11):

$j_{v, 1}=i \omega \eta_{1} \frac{L_{0,1}}{\kappa} u_{f, 1}=\frac{\omega^{2} \rho_{f, 1} L_{0,1}}{1+i \omega g_{0,1} \kappa \eta_{1}^{-1}} U_{s, 1} e^{i\left(\lambda_{1} z+\omega t\right)}=J_{1}^{\mathrm{wt}} e^{i\left(\lambda_{1}\left(z-z_{\mathrm{wt}}\right)+\omega t\right)}$,

where $J_{1}^{\mathrm{wt}}$ is the current density in medium 1 at the water table. In the same way we can obtain the current density in medium 2

$j_{v, 2}=i \omega \eta_{2} \frac{L_{0,2}}{\kappa} u_{f, 2}=\frac{\omega^{2} \rho_{f, 2} L_{0,2}}{1+i \omega g_{0,2} \kappa \eta_{2}^{-1}} U_{s, 2} e^{i\left(\lambda_{2}\left(z-z_{\mathrm{wt}}\right)+\omega t\right)}=J_{2}^{\mathrm{wt}} e^{i\left(\lambda_{2}\left(z-z_{\mathrm{wt}}\right)+\omega t\right)}$,

where $J_{2}^{\mathrm{wt}}$ is the current density in medium 2 at the water table. Note that, being the water content profile discontinuous at $z=z_{\mathrm{wt}}$, the relative fluid solid displacement will be discontinuous, and so will be the current density. Note that the jump in the current density $j_{v}$ is given by the difference between $J_{2}^{\mathrm{wt}}$ and $J_{1}^{\mathrm{wt}}$. From eqs (20) and (21) this is

$J_{2}^{\mathrm{wt}}-J_{1}^{\mathrm{wt}}=\left(\frac{\rho_{f, 2} L_{0,2}}{1+i \omega g_{0,2} \kappa \eta_{2}^{-1}}-\frac{\rho_{f, 1} L_{0,1}}{1+i \omega g_{0,1} \kappa \eta_{1}^{-1}}\right) \omega^{2} U_{s, 1}$.

This difference gives a measure of the jump in the amplitude of the current density at the water table and its value is mainly controlled by the jump in water saturation, which in turn produces jumps in $\rho_{f}$ and $L_{0}$ across the interface. Eq. (22) will be useful to interpret the electric and magnetic field behaviour in terms of the saturation contrast and the physical parameters of the medium.

\subsection{Electric and magnetic fields}

In this section, the influence of the current density as a source of electromagnetic fields is explored. Taking the first derivative with respect to $z$ in eq. (12) and replacing the resulting expression in eq. (11), the following non-homogeneous Helmholtz equation is obtained for the 
electric field $E$ :

$\frac{d^{2} E}{d z^{2}}+k^{2} E=i \omega \mu_{0} j_{v}$,

being $k=\sqrt{-i \omega \mu_{0} \sigma}$ the wavenumber. Replacing eqs (20) and (21) in eq. (23) we get:

$\frac{d^{2} E}{d z^{2}}+k_{1}^{2} E=i \omega \mu_{0} J_{1}^{\mathrm{wt}} e^{i \lambda_{1}\left(z-z_{\mathrm{wt}}\right)}, \quad 0 \leq z<z_{\mathrm{wt}}$,

$\frac{d^{2} E}{d z^{2}}+k_{2}^{2} E=i \omega \mu_{0} J_{2}^{\mathrm{wt}} e^{i \lambda_{2}\left(z-z_{\mathrm{wt}}\right)}, \quad z>z_{\mathrm{wt}}$.

Calling $\mathcal{L}_{*}$ the differential linear operator

$\mathcal{L}_{*}=\frac{d^{2}}{d z^{2}}+k_{*}^{2}$,

where $*$ denotes the medium ( 1 or 2 ), and calling

$\psi_{*}(z, \omega)=i \omega \mu_{0} J_{*}^{\mathrm{wt}} e^{i \lambda_{*}\left(z-z_{\mathrm{wt}}\right)}$,

then, eq. (24) can be expressed as:

$\mathcal{L}_{1}(E)=\psi_{1}(z, \omega)$;

it is straightforward to verify that

$E_{1, p}(z, \omega)=-\frac{k_{1}^{2} J_{1}^{\mathrm{wt}} e^{i \lambda_{1}\left(z-z_{\mathrm{wt}}\right)}}{\left(k_{1}^{2}-\lambda_{1}^{2}\right) \sigma_{1}}$

is a particular solution to it. On the other hand, the general solution for the homogeneous equation $\mathcal{L}_{1}(E)=0$ is given by:

$E_{1, \operatorname{hom}}(z, \omega)=A_{1} e^{-i k_{1} z}+B_{1} e^{i k_{1} z}$,

where $A_{1}$ and $B_{1}$ are frequency-dependent complex coefficients. Then, the complete solution in medium 1 can be written by adding both homogeneous and particular solutions

$E(z, \omega)=A_{1} e^{-i k_{1} z}+B_{1} e^{i k_{1} z}-\frac{k_{1}^{2} J_{1}^{\mathrm{wt}} e^{i \lambda_{1}\left(z-z_{\mathrm{wt}}\right)}}{\left(k_{1}^{2}-\lambda_{1}^{2}\right) \sigma_{1}}, \quad 0 \leq z<z_{\mathrm{wt}}$.

Following the same procedure, the solution for the electric field in medium 2 can be obtained:

$E(z, \omega)=A_{2} e^{-i k_{2} z}+B_{2} e^{i k_{2} z}-\frac{k_{2}^{2} J_{2}^{\mathrm{wt}} e^{i \lambda_{2}\left(z-z_{\mathrm{wt}}\right)}}{\left(k_{2}^{2}-\lambda_{2}^{2}\right) \sigma_{2}}, \quad z>z_{\mathrm{wt}}$.

The magnetic field $H$ can be derived from $E$ using eq. (12), leading to

$$
\begin{aligned}
& H(z, \omega)=\frac{k_{1}}{\omega \mu_{0}} A_{1} e^{-i k_{1} z}-\frac{k_{1}}{\omega \mu_{0}} B_{1} e^{i k_{1} z}-\frac{i \lambda_{1} J_{1}^{\mathrm{wt}} e^{i \lambda_{1}\left(z-z_{\mathrm{wt}}\right)}}{k_{1}^{2}-\lambda_{1}^{2}}, \quad 0 \leq z<z_{\mathrm{wt}}, \\
& H(z, \omega)=\frac{k_{2}}{\omega \mu_{0}} A_{2} e^{-i k_{2} z}-\frac{k_{2}}{\omega \mu_{0}} B_{2} e^{i k_{2} z}-\frac{i \lambda_{2} J_{2}^{\mathrm{wt}} e^{i \lambda_{2}\left(z-z_{\mathrm{wt}}\right)}}{k_{2}^{2}-\lambda_{2}^{2}}, \quad z>z_{\mathrm{wt}} .
\end{aligned}
$$

It is important to remark at this point that both fields are constituted by the superposition of two distinct responses. The first two terms in eqs (31)-(34), which are linked to the general solution of the homogeneous equation $\mathcal{L}_{*}(E)=0$ are perturbations travelling at velocities given by $\omega /|\operatorname{Re}(k)|$, that is, the velocity at which the electromagnetic signal travels in the soil. As it is well known, these perturbations are attributed to responses originated at interfaces between two distinct media, the so called IRs. The third terms, which are linked to the particular solutions of $\mathcal{L}_{*}(E)=\psi_{*}(z, \omega)$, can be clearly identified with the coseismic response, travelling at the same velocity $\omega /|\operatorname{Re}(\lambda)|$ as the solid and fluid displacements. Observing the magnetic coseismic field (H-Cos), for example in eq. (33), it is clear that its origin is the current density $J_{1}^{\mathrm{wt}}$ arisen from the shearing motion of the rock matrix, which in turn drags the viscous fluid and therefore the electric charges in it. The origin of the E-Cos is, again as can be seen in the equations, the time derivative of the magnetic field, that is, the electric field is induced, through Faraday's law, by the time variation of the magnetic field. This behaviour of the fields was conceptually discussed in, e.g. Haartsen \& Pride (1997), Zyserman et al. (2015), Munch \& Zyserman (2016). On the other hand, the situation is different for the coseismic electric field located within compressional waves, because it is created by accumulation and depletion of electrokinetic charge in regions of dilation and compression (Pride \& Haartsen 1996; Haines et al. 2007; Jouniaux \& Zyserman 2016). The unknown coefficients $A_{1}, B_{1}, A_{2}$ and $B_{2}$ should be obtained by imposing conditions on the interface of both media at $z=z_{\mathrm{wt}}$, and at the boundaries of the system $(z=0$ and $z \rightarrow)$ as it is shown below. In order to avoid the divergence of the electromagnetic fields when $z \rightarrow$, we set $B_{2}=0$ (for $k_{2}$ we choose the square root with negative imaginary part). If the Earth's surface is in contact with air, and if we assume that the air is an insulator, then the amplitude 
of the electric field must be constant for $z \leq 0$ in order to avoid its divergence when $z \rightarrow-$, and in virtue of eq. (12) $H=0$ for $z<0$ (see Appendix B). Given that the magnetic field should be continuous at $z=0$ (Appendix C shows the continuity of $H$ at $z=0$ and $z=z_{\mathrm{wt}}$, see Fig. C1), we can write:

$0=A_{1}-B_{1}+\frac{\lambda_{1} k_{1} J_{0}}{\left(k_{1}^{2}-\lambda_{1}^{2}\right) \sigma_{1}}$

where $J_{0}=J_{1}^{\mathrm{wt}} e^{-i \lambda_{1} z_{\mathrm{wt}}}$ is the current density at the surface. Both the electric and magnetic fields should be continuous at the water table (see Appendix C). The continuity condition for both fields at the water table can be, respectively, stated as follows:

$A_{1} e^{-i k_{1} z_{\mathrm{wt}}}+B_{1} e^{i k_{1} z_{\mathrm{wt}}}-\frac{k_{1}^{2} J_{1}^{\mathrm{wt}}}{\left(k_{1}^{2}-\lambda_{1}^{2}\right) \sigma_{1}}=A_{2} e^{-i k_{2} z_{\mathrm{wt}}}-\frac{k_{2}^{2} J_{2}^{\mathrm{wt}}}{\left(k_{2}^{2}-\lambda_{2}^{2}\right) \sigma_{2}}$,

$k_{1}\left(A_{1} e^{-i k_{1} z_{\mathrm{wt}}}-B_{1} e^{i k_{1} z_{\mathrm{wt}}}\right)+\frac{\lambda_{1} k_{1}^{2} J_{1}^{\mathrm{wt}}}{\left(k_{1}^{2}-\lambda_{1}^{2}\right) \sigma_{1}}=k_{2} A_{2} e^{-i k_{2} z_{\mathrm{wt}}}+\frac{\lambda_{2} k_{2}^{2} J_{2}^{\mathrm{wt}}}{\left(k_{2}^{2}-\lambda_{2}^{2}\right) \sigma_{2}}$.

The system of eqs (35)-(37) completely determine the values for the three coefficients $A_{1}, B_{1}$ and $A_{2}$ :

$A_{1}=-\frac{1}{2} \frac{\left[\frac{\lambda_{1} k_{1}\left(k_{1}+k_{2}\right) e^{i k_{1} z_{\mathrm{wt}} J_{0}}}{\left(k_{1}^{2}-\lambda_{1}^{2}\right) \sigma_{1}}-\frac{k_{1}^{2}\left(k_{2}+\lambda_{1}\right) J_{1}^{\mathrm{wt}}}{\left(k_{1}^{2}-\lambda_{1}^{2}\right) \sigma_{1}}+\frac{k_{2}^{2}\left(k_{2}+\lambda_{2}\right) J_{2}^{\mathrm{wt}}}{\left(k_{2}^{2}-\lambda_{2}^{2}\right) \sigma_{2}}\right]}{k_{1} \sinh \left(i k_{1} z_{\mathrm{wt}}\right)+k_{2} \cosh \left(i k_{1} z_{\mathrm{wt}}\right)}$,

$B_{1}=-\frac{1}{2} \frac{\left[\frac{\lambda_{1} k_{1}\left(k_{1}-k_{2}\right) e^{-i k_{1} z_{\mathrm{wt}} J_{0}}}{\left(k_{1}^{2}-\lambda_{1}^{2}\right) \sigma_{1}}-\frac{k_{1}^{2}\left(k_{2}+\lambda_{1}\right) J_{1}^{\mathrm{wt}}}{\left(k_{1}^{2}-\lambda_{1}^{2}\right) \sigma_{1}}+\frac{k_{2}^{2}\left(k_{2}+\lambda_{2}\right) J_{2}^{\mathrm{wt}}}{\left(k_{2}^{2}-\lambda_{2}^{2}\right) \sigma_{2}}\right]}{k_{1} \sinh \left(i k_{1} z_{\mathrm{wt}}\right)+k_{2} \cosh \left(i k_{1} z_{\mathrm{wt}}\right)}$,

$A_{2}=-\frac{\left[\frac{\lambda_{1} k_{1}^{2} J_{0}}{\left(k_{1}^{2}-\lambda_{1}^{2}\right) \sigma_{1}}-\frac{k_{1}^{2}\left(\lambda_{1} \cosh \left(i k_{1} z_{\mathrm{wt}}\right)-k_{1} \sinh \left(i k_{1} z_{\mathrm{wt}}\right)\right) J_{1}^{\mathrm{wt}}}{\left(k_{1}^{2}-\lambda_{1}^{2}\right) \sigma_{1}}+\frac{k_{2}^{2}\left(\lambda_{2} \cosh \left(i k_{1} z_{\mathrm{wt}}\right)-k_{1} \sinh \left(i k_{1} z_{\mathrm{wt}}\right)\right) J_{2}^{\mathrm{wt}}}{\left(k_{2}^{2}-\lambda_{2}^{2}\right) \sigma_{2}}\right]}{k_{1} \sinh \left(i k_{1} z_{\mathrm{wt}}\right)+k_{2} \cosh \left(i k_{1} z_{\mathrm{wt}}\right)} e^{i k_{2} z_{\mathrm{wt}}}$,

with which we have the complete solutions for the electric and magnetic fields. Once the fields $E(z, \omega)$ and $H(z, \omega)$ are known for a given depth $z$ below the surface, the time variation of these fields at that depth is obtained by the inverse Fourier transform. In the following section, hypothetical examples are proposed to analyse the electromagnetic responses predicted by the derived analytical solutions.

\subsection{Approximate electromagnetic fields}

Although the analytical expressions for the fields (eqs 31-34) seem to be quite simple, the involved coefficients $A_{1}, B_{1}$ and $A_{2}$ are not. However, under reasonable assumptions it is possible to find a simple approximation to the solution suitable to perform an analysis of the SHTE response of the vadose zone.

Consider the ratio $\left(k_{*} / \lambda_{*}\right)^{2}$, given by eq. (16) and the definition of the wavenumber $k_{*}$ :

$$
\left(\frac{k_{*}}{\lambda_{*}}\right)^{2}=\frac{i \mu \sigma_{*} G}{\rho_{b, *}-\frac{\rho_{f, *}^{2}}{g_{0, *}-i \eta_{*} /(\kappa \omega)}} .
$$

We have computed this quotient for five different soil textures and for a wide range of seismic frequencies, observing that its absolute value is not bigger than $1 \times 10^{-4}$, so we can safely consider it as a very small quantity. By doing this, it is possible to make some approximations to simplify the expressions of the fields. Neglecting the ratio $\left(k_{*} / \lambda_{*}\right)^{2}$ against $k_{*} / \lambda_{*}$ and assuming that $e^{ \pm i k_{1} z} \simeq 1$, which is a very good approximation when $z$ is not higher than $10^{2} \mathrm{~m}$, then the electric and magnetic fields can be approximated by the following simplified expressions:

$E(z, \omega) \simeq \frac{\omega \mu_{0}}{k_{2}}\left[\frac{J_{0}}{i \lambda_{1}}-\frac{J_{1}^{\mathrm{wt}}}{i \lambda_{1}}+\frac{J_{2}^{\mathrm{wt}}}{i \lambda_{2}}\right], \quad z \geq 0$,

$H(z, \omega) \simeq \frac{J_{0}}{i \lambda_{1}}-\frac{J_{1}^{\mathrm{wt}} e^{i \lambda_{1}\left(z-z_{\mathrm{wt}}\right)}}{i \lambda_{1}}, \quad 0 \leq z<z_{\mathrm{wt}}$,

$H(z, \omega) \simeq\left[\frac{J_{0}}{i \lambda_{1}}-\frac{J_{1}^{\mathrm{wt}}}{i \lambda_{1}}+\frac{J_{2}^{\mathrm{wt}}}{i \lambda_{2}}\right]-\frac{J_{2}^{\mathrm{wt}} e^{i \lambda_{2}\left(z-z_{\mathrm{wt}}\right)}}{i \lambda_{2}}, \quad z>z_{\mathrm{wt}}$

Note that these last expressions are simpler than those of the exact solutions, particularly the terms corresponding to the interface response; the complex coefficients $A_{1}, B_{1}$ and $A_{2}$ appearing in the exact solution are no longer present in the approximate solution. The three terms between brackets in eqs (42) and (44) together with the first term in eq. (43) constitute the interfacial response of the approximate solution, meanwhile the last terms in eqs (43) and (44) give the coseismic response. In the following we analyse the electromagnetic responses predicted by both the exact and the approximate solutions. It is shown that the latter result is a very good approximation to the exact solution, and due to the simplicity of the mathematical expressions of the fields, they are convenient to analyse the seismoelectrical response of the vadose zone. 

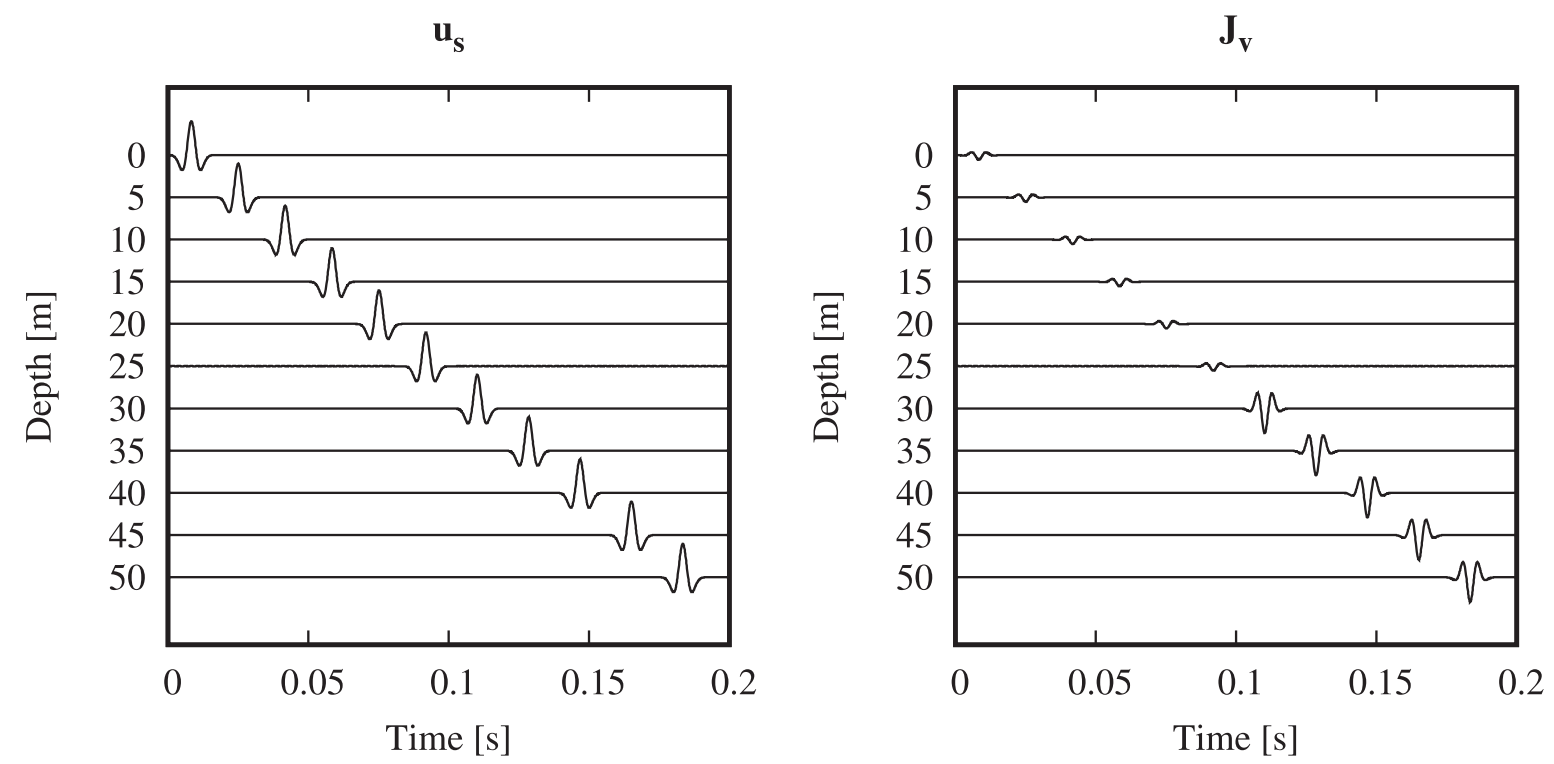

Figure 2. Time variation of the solid displacement (left) and electrical current density (right) registered at 11 receivers. The distance between receivers is $5 \mathrm{~m}$. Above $25 \mathrm{~m}$ depth the water saturation is 0.32 , and below $25 \mathrm{~m}$ depth the medium is fully saturated.

\section{RESULTS AND DISCUSSION}

We describe here the coseismic and interfacial response of the water table induced by the propagation of an $S H$ wave produced by a shear source at the surface. We first show the displacement and electric current density, then the coseismic and interfacial signals of both the electric and magnetic fields. We analyse the amplitude of these electromagnetic conversions, and the effect produced by changes in water saturation, porosity, fluid viscosity and density.

\subsection{Seismic waves and viscous current density analysis}

To illustrate the field responses predicted by the derived analytical solution we compute, as explained before, the physical parameters given in 'Theoretical Background' section using the basic parameters listed in Table 1. Both the porous matrix properties and the effective properties of the media are computed for a silt loam, using the same soil parameters as in Zyserman et al. (2017a). The water table is assumed to be located at $25 \mathrm{~m}$ below the surface, and as the time signature of the seismic source $f^{s}(t)$ we use a Ricker wavelet with peak frequency of $120 \mathrm{~Hz}$, the peak amplitude for the Ricker wavelet (located at $t=8 \times 10^{-3} \mathrm{~s}$ ) is set so that the amplitude of the solid displacement at the surface is $U_{s, 1}=1 \mathrm{~m}$. Note that this is not a realistic value for the solid displacement but it is appropriated for a qualitative analysis. The vadose zone water saturation is taken to be the mean value of the saturation profile defined by the van Genuchten model. For the silt loam (see Zyserman et al. 2017a) with the water table located at the same depth as above, the mean saturation value is $S_{\mathrm{w}}=0.32$.

The time variation of the solid displacement and the current density is plotted in Fig. 2 for 11 different depths measured from the surface down to $50 \mathrm{~m}$ depth. For visualization purposes, the fields were scaled by multiplying the $u_{s}$ field and the $J_{v}$ field by 4 and 1.5 , respectively, so that the wavelets have almost the same amplitudes; we proceed in this way because the purpose of Fig. 2 is to analyse the time signatures of the fields but not the amplitudes, which are studied in the following sections. As it can be seen, the solid displacement $u_{\mathrm{s}}$ induced by the source at the surface travels downwards with fairly constant seismic velocity and amplitude. The current density $j_{v}$ follows the same time dependence as $u_{\mathrm{s}}$ but with a sharp increase of amplitude when the water table is reached at $25 \mathrm{~m}$ depth. This jump in the current density was expected given the sharp contrast in water saturation. This contrast is the main contribution to the variation of the current density across the water table (see eq. 22).

\subsection{Coseismic field analysis}

The coseismic responses of the electric and magnetic fields are shown in Fig. 3 (left-hand panels). These fields are obtained by computing the third term in eqs (31) and (34), respectively. As we did in the previous figure, for visualization purposes the fields were scaled by multiplying the E-Cos field and the H-Cos field by 25000 and 8, respectively; again, the scaling does not affect the behaviour in time of the traces. The travelling seismic wave induces a relative motion between the rock matrix and the water due to the non-zero viscosity of the latter, which produces an electric current density. This current density induces the coseismic magnetic field. The coseismic field travels with the seismic wave and therefore shows the same propagation pattern with depth. The coseismic responses of the magnetic field H-Cos and of the electric field E-Cos show the same behaviour as the current density, with higher amplitudes below the water table. Indeed the amplitudes of 
E-Cos

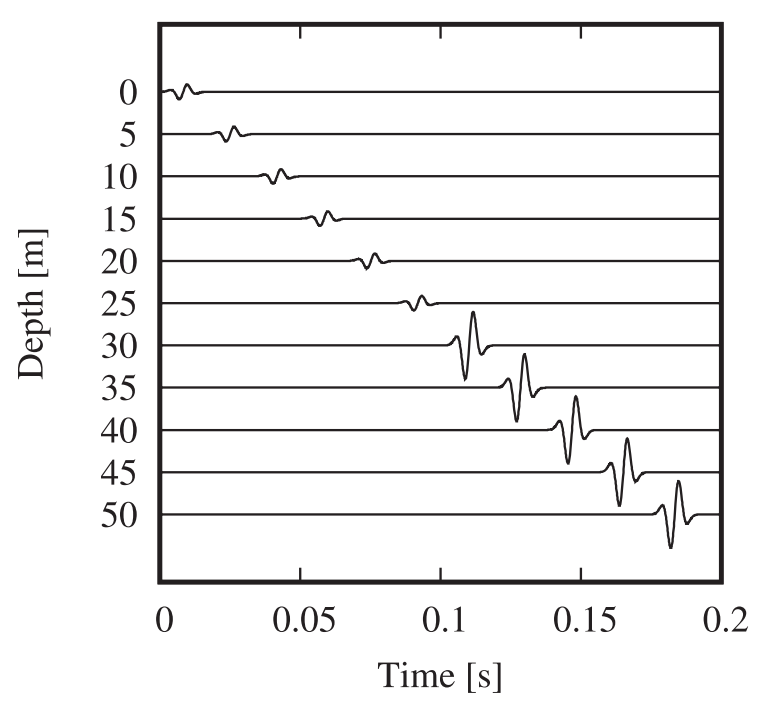

H-Cos

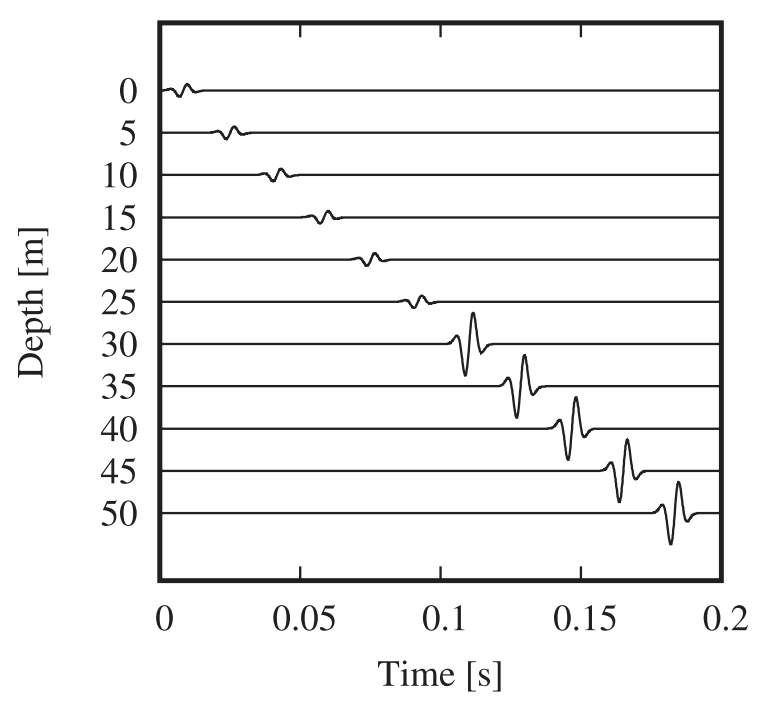

E-IR

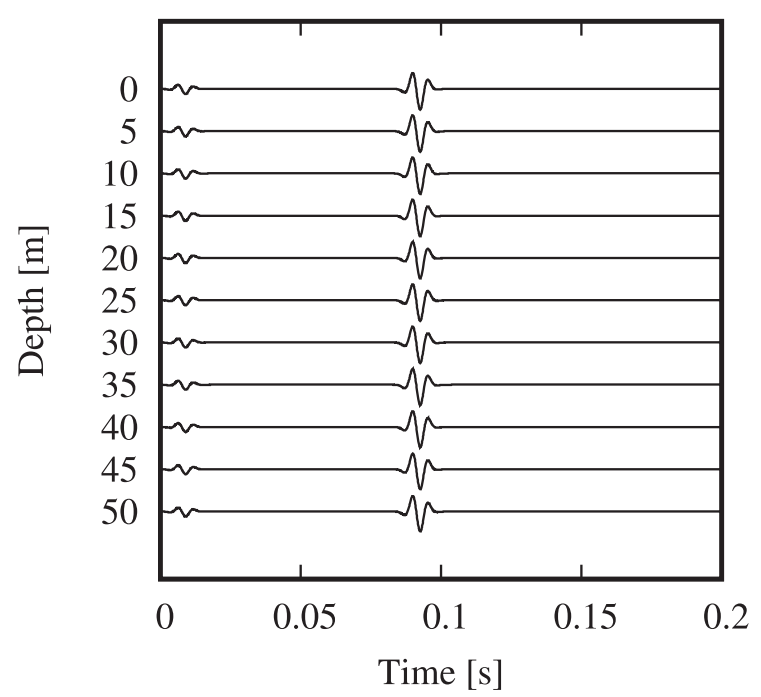

H-IR

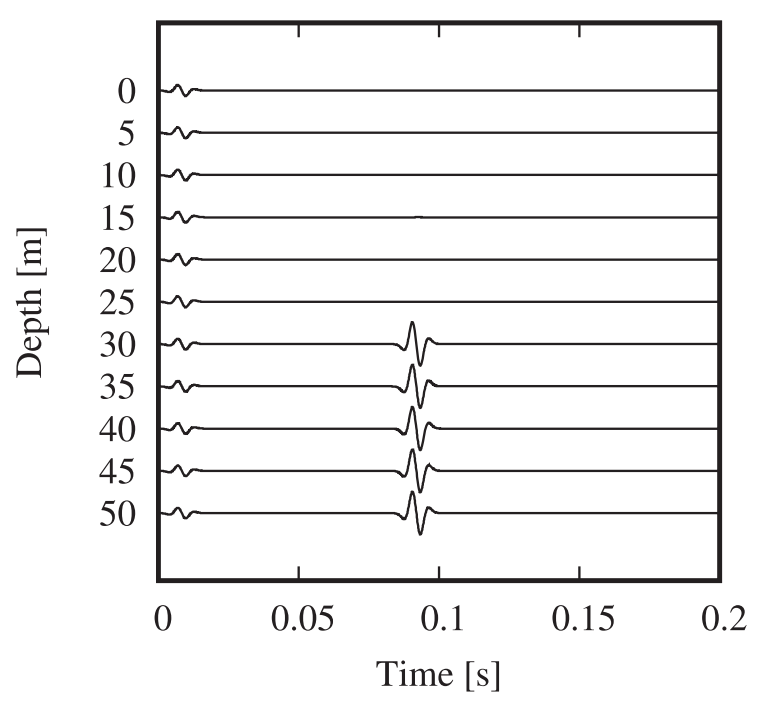

Figure 3. Time variation of the coseismic (left) and interfacial (right) responses of both electric (top) and magnetic (bottom) fields registered at 11 receivers. The distance between receivers is $5 \mathrm{~m}$. Above $25 \mathrm{~m}$ depth the water saturation is 0.32 , and below $25 \mathrm{~m}$ depth the medium is fully saturated.

the coseismic fields are proportional to the current density (see the third term in eqs 31-34, respectively). We recall here that the E-Cos is originated in the time dependence of the H-Cos, through Faraday's law, eq. (12) (Zyserman et al. 2017a).

\subsection{Interfacial responses analysis}

The interfacial responses are shown in Fig. 3 (right-hand panels) and are given by the first two terms of eqs (31)-(34). The fields were scaled by multiplying the E-IR field and the H-IR field by 8 and 7, respectively. As it can be seen, two distinct events occurring at two different times are registered simultaneously at all depths (in the case of H-IR, the second event is not distinguishable over the water table because its amplitude is negligible). The first one has its peak amplitude at the same time as the seismic source $\left(8 \times 10^{-3} \mathrm{~s}\right)$, and for this reason is identified as an IR originated at the surface. This IR is attributed to the jump in the current density at the surface (note that the current density over the surface is zero, and at the surface it takes the value $J_{0}$ ). Although there is numerical evidence of the existence of this IR (Zyserman et al. 2015; Munch \& Zyserman 2016), this is the first time that its existence is predicted analytically. As it also can be seen in the figure, the interface response of the surface is registered for both the electric and magnetic fields, and depicts the same amplitude for all the receivers located both over and below the water table. A second event is registered at a time that is compatible with the time needed by the seismic wave to reach the water table, located at $25 \mathrm{~m}$ depth, which is $\simeq 0.083 \mathrm{~s}$ for a seismic velocity of $300 \mathrm{~m} \mathrm{~s}^{-1}$. Once the $S H$ seismic wave reaches the water table, there is a discontinuity in the electric current density and therefore a strong amplitude variation in the magnetic coseismic field 

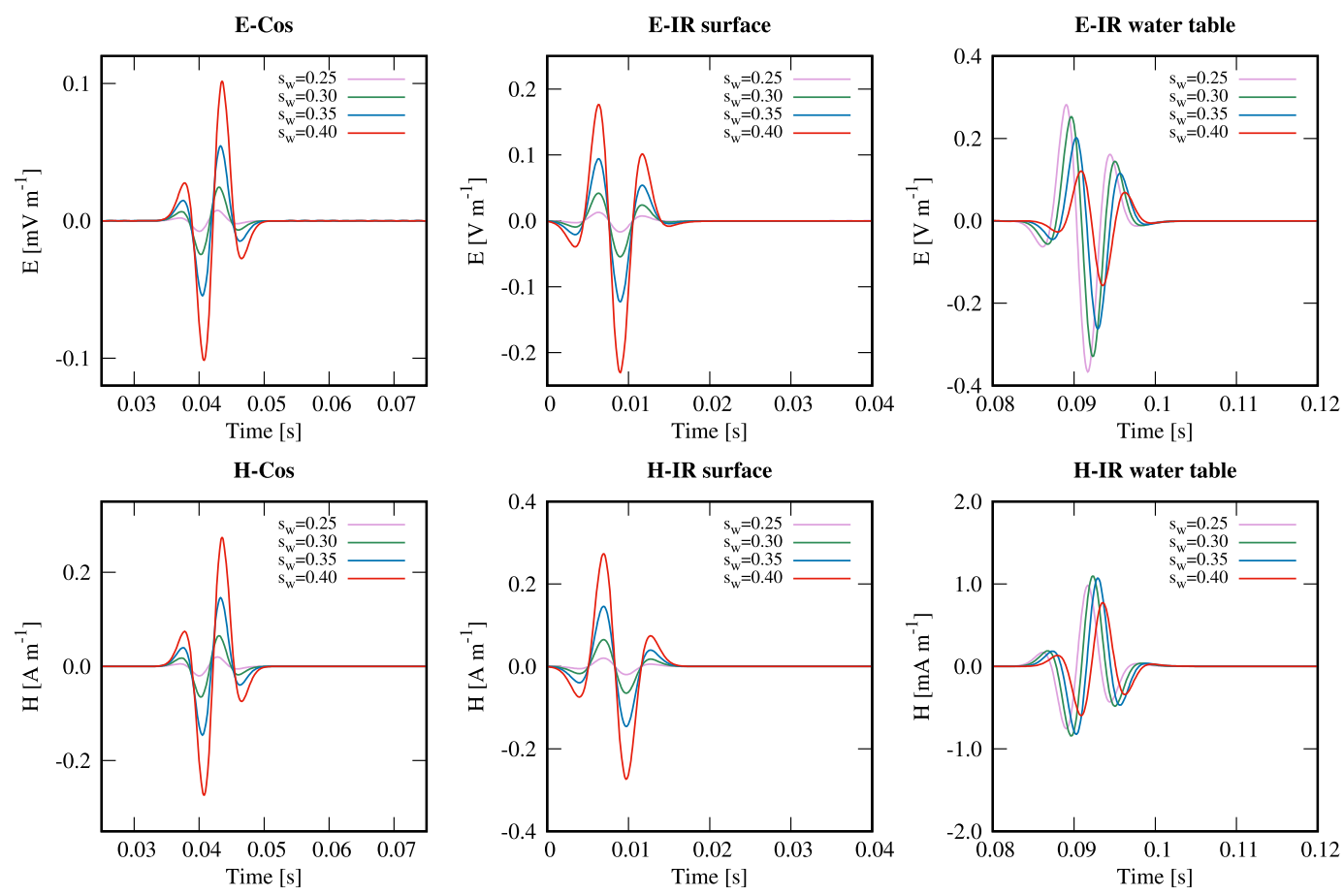

Figure 4. Time variation of the coseismic response (left), interfacial response of the surface (center) and interfacial response of the water table (right) of both electric (top) and magnetic (bottom) fields registered at $z=10 \mathrm{~m}$ (15 m over the water table) for the four different saturation values considered in this study.

$\mathrm{H}-\mathrm{Cos}$. Note, however, that the total field $\mathrm{H}$ is continuous across the water table, as we proved in Appendix C. The jump in the current density leads to the magnetic interfacial response H-IR, and to the electric interfacial response E-IR, which travel in the porous medium at a velocity much larger than the seismic velocity and arrive at (nearly) the same time at all depths. Note that in this case, the interface response of the electric field preserves its amplitude across the water table, contrary to what we have just described happens with the magnetic field $\mathrm{H}$. This is a consequence of the continuity of the tangential component of the electric field at any interface. The behaviour for the IRs of both fields confirms the novel numerical results we obtained in our previous work (Zyserman et al. 2017a). They are different from what is known from the analysis of seismoelectromagnetic conversions induced by compressional waves: Warden et al. (2013) and (Bordes et al. 2015; Peng et al. 2017) predicted through numerical modelling and observed on experiments that in this case the IRs have a very small amplitude compared to the coseismic signals.

At this point, we want to mention that in most theoretical and numerical scenarios present in the literature is affirmed that the coseismic electric field accompanying the $P$ wave is larger than that of the $S$ wave. However, a numerical study of Gao et al. (2017), based on a relation for the transfer functions for both $P$ and $S$ waves deduced previously (Gao \& Hu 2010), showed that there are indeed realistic situations in which the $S$-wave coseismic electric field can be of the same order of magnitude as that of the $P$ wave. However, this is of no concern to the relative amplitudes of the IRs generated by the $P$ - and $S$-seismic waves. The origin of the IRs, associated to different physical processes (electric charge accumulation in the first case, electric currents in the second one) and how they depend on the geometry and physical parameters of the system under research, is not completely known, fundamentally for the $S H$-wave IRs. In this regard, a point that must not be overlooked is that the IRs generated by the arrival of $P$ waves to an interface $\left(\mathrm{E}_{P}-\mathrm{IR}\right)$ present not only the problem that the amplitude of the coseismic signal is usually stronger than the $\mathrm{E}_{P}-\mathrm{IR}$, but they are also recorded simultaneously for usual acquisition geometries, what makes their individualization very difficult. This situation persists even in the case in which the $\mathrm{E}_{P}$-IR be stronger than those generated by $S H$ waves $\left(\mathrm{E}_{S H}-\mathrm{IR}\right)$. Therefore, we think that the signal-to-noise ratio E-IR/E-Cos becomes more relevant than the relative amplitude between the $\mathrm{E}_{P}-\mathrm{IR}$ and the $\mathrm{E}_{S H}$-IR fields when evaluating the possibility of individualizing the interface response. The difficulty in getting a meaningful $\mathrm{E}_{P}$-IR from a surface-to-surface recording is well known, and it could be overcome by setting other source and receivers geometries, e.g. surface-to-borehole, as it has been proposed in the literature (Dupuis et al. 2009). On the other hand, the fact that for $S H$-wave sources the E-Cos signal is much lower than the E-IR leads in principle to a much better signal-to-noise ratio even for the surface-to-surface geometry to detect the interfacial response.

\subsection{Water saturation dependence of the amplitude of the electromagnetic responses}

We explore the dependence of the electromagnetic responses on the saturation $S_{\mathrm{w}}$ of medium 1 . The coseismic and interfacial responses of the surface and the water table, for water saturation between 25 per cent to 40 per cent computed at $z=10 \mathrm{~m}$ ( $15 \mathrm{~m}$ over the water table) and $z=40 \mathrm{~m}$ ( 15 below the water table) are shown in Figs 4 and 5, respectively. As it can be seen in Fig. 4, the amplitude of the electric coseismic 

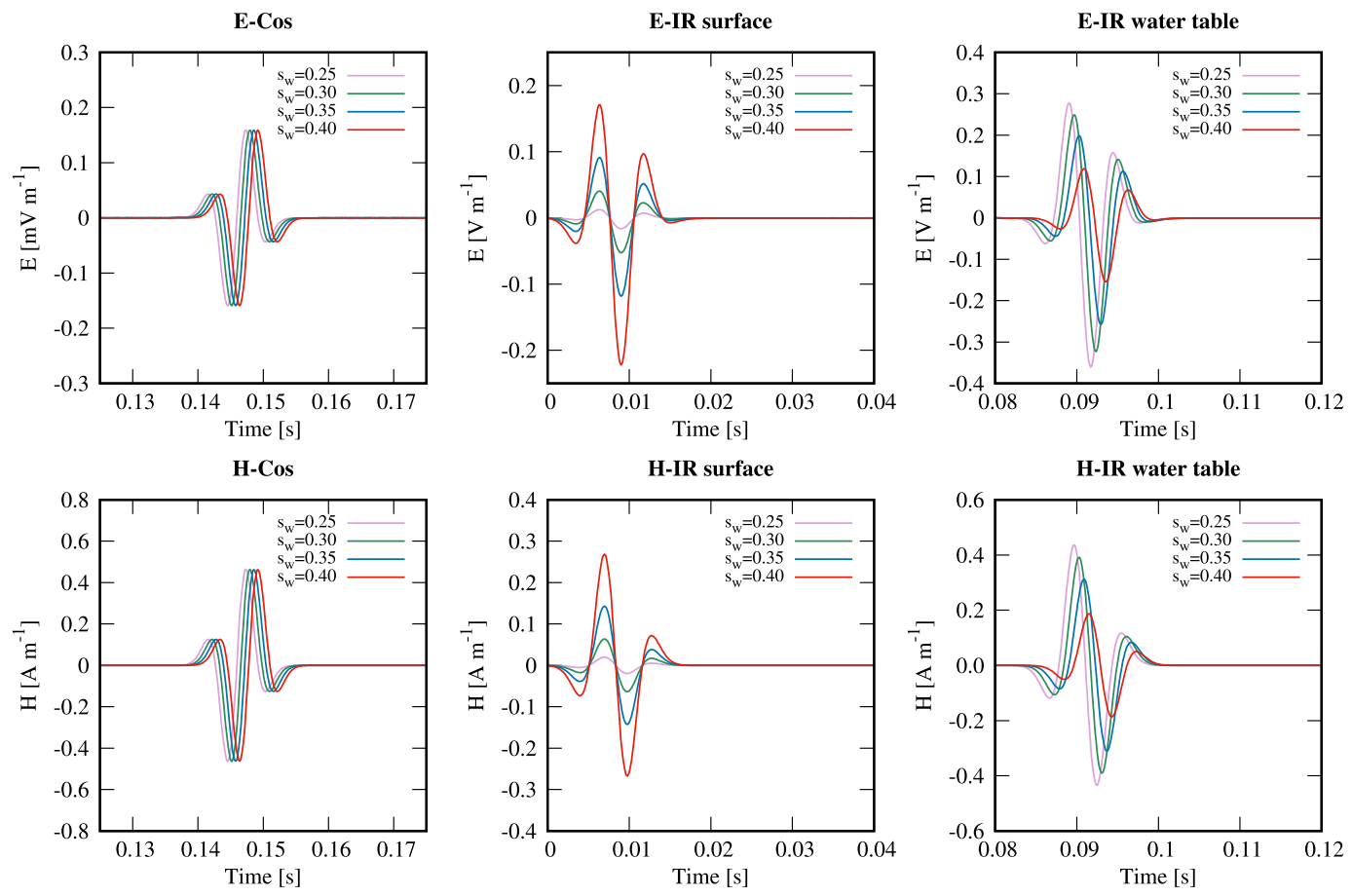

Figure 5. Time variation of the coseismic response (left), interfacial response of the surface (center) and interfacial response of the water table (right) of both electric (top) and magnetic (bottom) fields registered at $z=40 \mathrm{~m}$ (15 m below the water table) for the four different saturation values considered in this study.

E-IR/E-Cos field at $\mathrm{z}=\mathbf{1 0} \mathrm{m}$

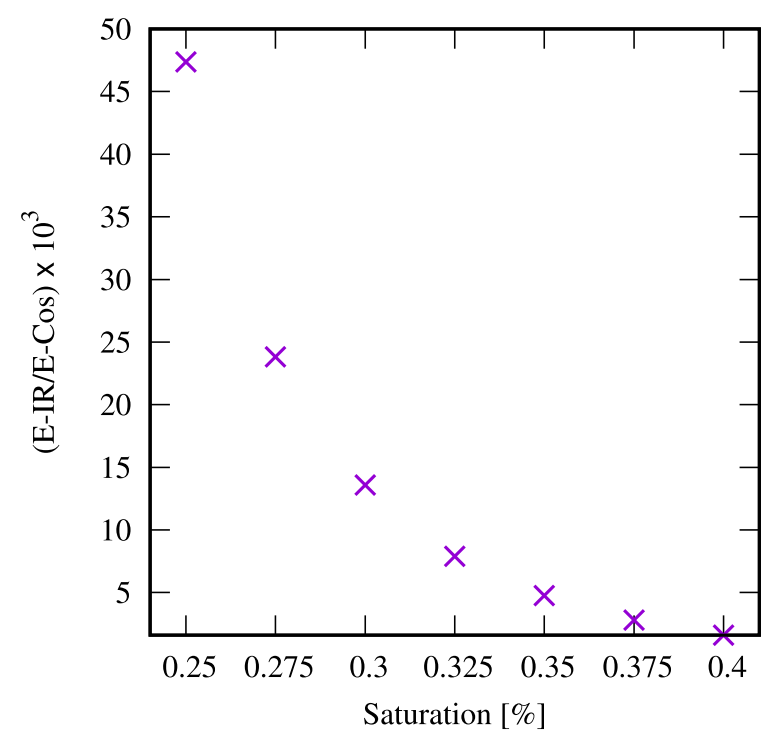

Figure 6. Ratio between the peak amplitudes of the electric interfacial response E-IR of the water table and coseismic response E-Cos computed at a receiver located $10 \mathrm{~m}$ below the surface and for seven different values of water saturation of the vadose zone.

field is in the range of $0.1 \mathrm{mV} \mathrm{m}^{-1}$ while the amplitude of both electric interfacial responses reaches amplitudes in the order of $0.2-0.4$ $\mathrm{V} \mathrm{m}^{-1}$. The amplitude of the coseismic electric field is decreasing with decreasing water saturation, and the same occurs to the surface E-IR, whereas the amplitude of the water table E-IR is increasing with decreasing water saturation. The water table E-IR is therefore several orders of magnitude higher than the E-Cos and can be detected at $10 \mathrm{~m}$ depth or at the surface, and is enhanced if the water saturation is decreased, from a ratio of 3 orders of magnitude at saturation 40 per cent to 4 orders of magnitude at saturation 25 per cent. This behaviour is well explained in Fig. 6, where the ratio of the peak amplitudes between IR of the water table and E-Cos responses of the electric field is plotted as a function of water saturation of the vadose zone. Still in the unsaturated zone, at $10 \mathrm{~m}$ depth the amplitudes of the magnetic coseismic field and the magnetic interfacial response of the surface are both in the range $0.1-0.3 \mathrm{~A} \mathrm{~m}^{-1}$, both fields are decreasing with decreasing water saturation. Although the magnetic interface response of the water table is present in the registers located above the water table, its amplitude 

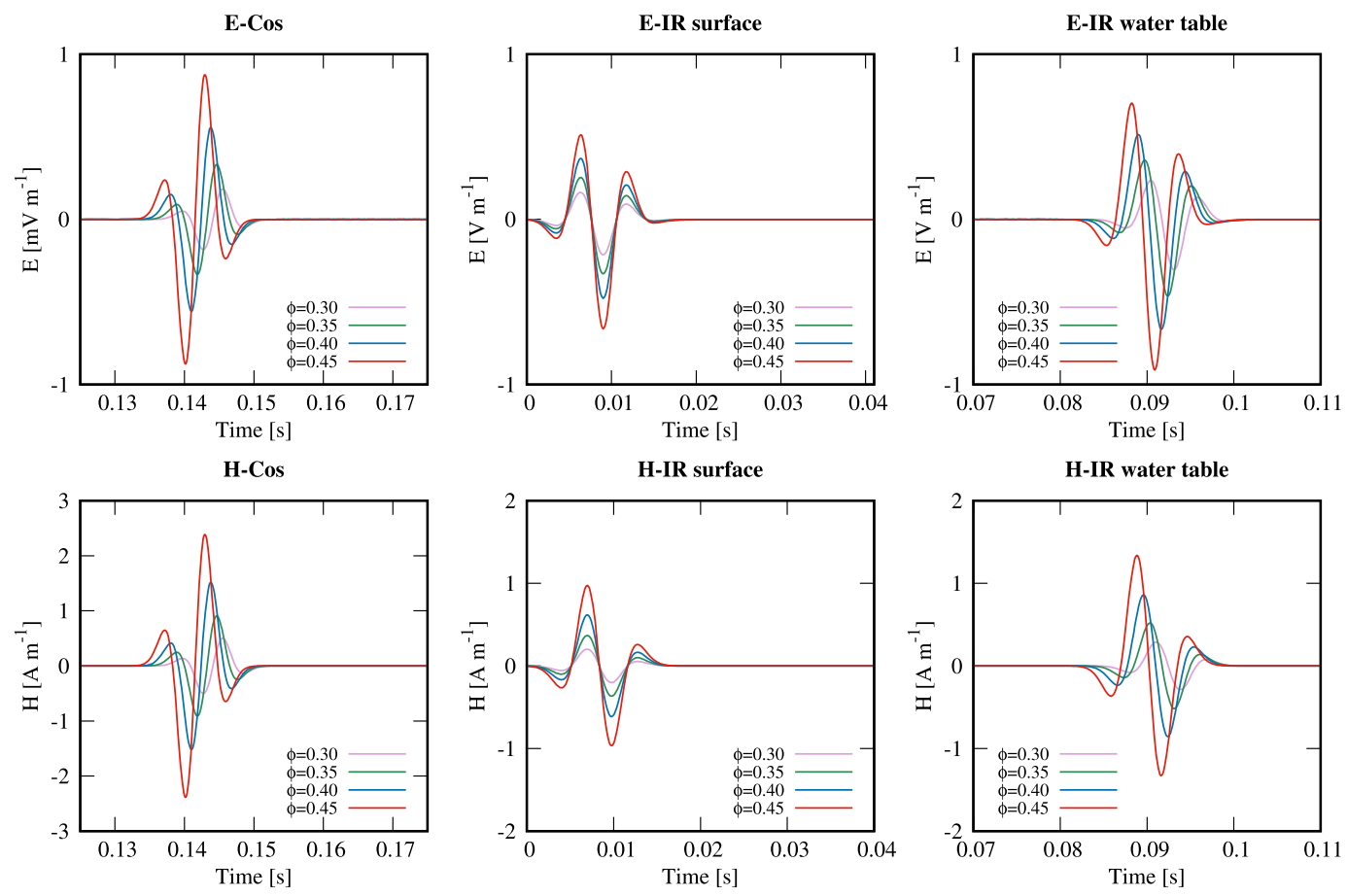

Figure 7. Time variation of the coseismic (left), interfacial response of the surface (center) and interfacial response of the water table (right) of both electric (top) and magnetic (bottom) fields registered at $z=40 \mathrm{~m}$ (15 m below the water table) for four different porosity values considered in this study.

is negligible, as can be seen from Fig. 4. The amplitudes for the H-IR of the water table are between 2 and 3 orders of magnitude lower, so that the ratio between the H-IR and H-Cos will not allow us to detect to H-IR.

In the saturated zone, at $40 \mathrm{~m}$ depth (see Fig. 5), the amplitude of the E-Cos is about $0.15 \mathrm{mV} \mathrm{m}^{-1}$ and the E-IR of the water table is about $0.1-0.3 \mathrm{~V} \mathrm{~m}^{-1}$, leading to a ratio between the E-IR over the E-Cos of about $667-2000$ for water saturations of 25-40 per cent, respectively. The E-IR from the water table could therefore be detected in the saturated zone. The amplitude of the coseismic magnetic field is about $0.5 \mathrm{~A}$ $\mathrm{m}^{-1}$ and the one of the H-IR of the water table is $0.2-0.4 \mathrm{~A} \mathrm{~m}^{-1}$ for water saturations of $25-40$ per cent. Therefore, the ratio between the H-IR and H-Cos is only 0.4 to 0.8 , and the H-IR will be hardly detected at depth. As it can be seen, the amplitude variation of E-IR and H-IR with water saturation depicts the same behaviour. However, the amplitudes of E-Cos and H-Cos remain constant [note in eqs (32) and (34) that the expressions for the coseismic fields in the saturated zone have no dependence on the saturation of the unsaturated zone]. Note that both for $z=10 \mathrm{~m}$ and $z=40 \mathrm{~m}$ the saturation variation induces a time shift that is present in the coseismic fields and in the interfacial response of the water table, but is not in the interfacial response of the surface. We attribute this shift to a decrease in the velocity of medium 1 with the increase of water saturation; this behaviour is observed from the shear wave velocity obtained from eq. (16) and has been experimentally observed in partially saturated rocks (Mochizuki 1982). It is important to remark that this analysis allows to conclude that a $S H$-wave source can induce an E-IR from the water table which can be detected at surface or at depth because it is several orders of magnitude larger than the E-Cos field. We observe that an increase of the water saturation contrast between the vadose zone and the saturated zone will produce an increase in the amplitude of the water table E-IR and H-IR together with a decrease of the amplitude of the surface E-IR and H-IR.

\subsection{Sensitive analysis}

We analyse here the effect of changes in porosity, fluid viscosity and density on the electromagnetic conversions. The analysis is performed on time traces of the electric and magnetic fields registered at one receiver. The results observed for other depths are qualitatively the same when analyzing the effect of these parameters. In this case we choose a receiver located below the water table $(z=40 \mathrm{~m})$ because all events can be visualized (remember that the amplitude of H-IR at the water table is almost negligible for receivers located over the water table).

The coseismic and interfacial responses for porosities between 0.30 and 0.45 computed at $z=40 \mathrm{~m}$ are shown in Fig. 7. Note that the changes in porosity produce both amplitude and time shift variations on the fields. Particularly, when the porosity is increased, the time shift of the events (with the exception of the IRs of the surface) is decreased. This is due to the small increase of seismic velocity produced when the porosity of the medium is increased; this behaviour is theoretically predicted by the shear wave velocity obtained from eq. (16) and has been experimentally observed in unconsolidated sands (Zimmer et al. 2007). It can also be observed in Fig. 7 that the amplitude of the electromagnetic fields shows a smooth increase with porosity that causes a higher fluid volume to be displaced, which in turn produces higher current densities. The former statement is based in an analysis of the behaviour of eq. (18) with porosity we performed but don't show here 

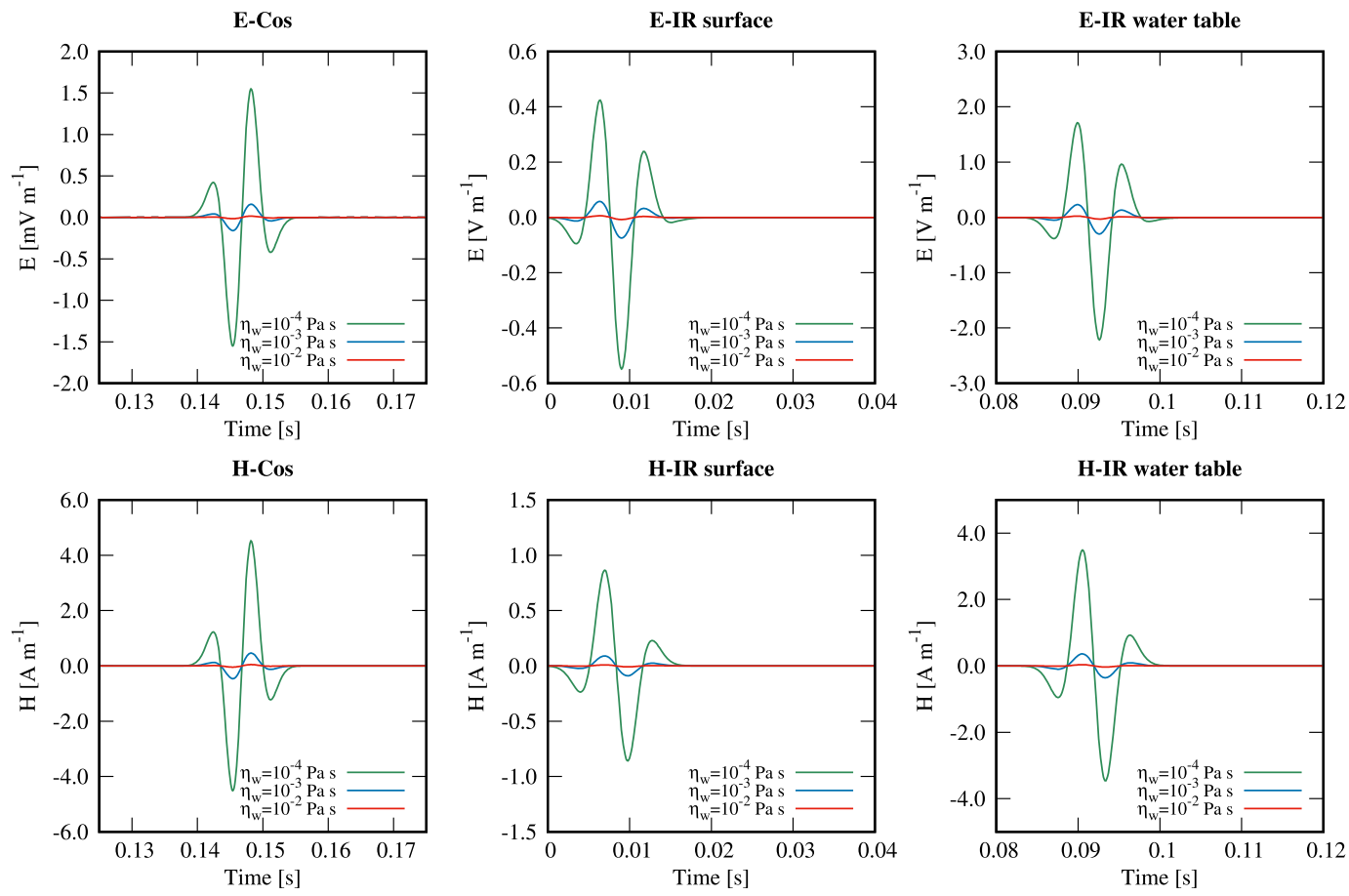

Figure 8. Time variation of the coseismic response (left), interfacial response of the surface (center) and interfacial response of the water table (right) of both electric (top) and magnetic (bottom) fields registered at $z=40 \mathrm{~m}$ (15 m below the water table) for three different viscosity values considered in this study.

for the sake of brevity. These higher current densities increase the amplitudes of the fields, explaining the amplitude variation showed by the fields when porosity is increased.

The impact of fluid viscosity variations on the electromagnetic fields is analysed by considering time traces of the electromagnetic fields registered at $z=40 \mathrm{~m}$ for three different values of fluid viscosity (see Fig. 8). As in the case of porosity variations considered before, we choose a receiver below the surface because all events are clear and the results obtained for other traces are qualitatively the same. It is worth to mention that the variation is introduced in the viscosity of water, i.e. $\beta_{\mathrm{w}}$. As it can be seen in Fig. 8 , the increase of viscosity produces a decrease in the amplitude of both fields, with no time shift present in the traces. This effect is attributed to energy dissipation, which is stronger for higher fluid viscosities; this fact can be observed from eq. (18); the real part of this expression gives the amplitude of the fluid displacement, the imaginary part accounts for the energy dissipation. Then, the amplitude of the current density induced by the shear wave is lower, which in turn produces a lower amplitude coseismic response and, therefore, smaller interface responses.

Finally, we analyse the effect of fluid density variations on the electromagnetic fields by considering time traces registered at $z=40 \mathrm{~m}$ for three different values of water density (see Fig. 9). Both amplitude and time shift variations are produced when the water density is changed, and this variations are present in all the events with the exception of the IR of the surface (only amplitude variations are shown by this event). The increase of water density produces an increase in the amplitude of both fields. Note that this amplitude increase is in the same order than the one observed for porosity changes. There is a shift in time as in the case of the porosity, but again it can be attributed to variations in the seismic velocity induced by the change in fluid density. The increase of water density produces a decrease of the seismic wave velocity (see eq. 16), which in turn produces a shift towards later times of the events registered, as can be seen in the figure. The amplitude variation, however, is mainly attributed to a variation on the current density, which depends on fluid density (the current density is higher for higher fluid densities), see eqs (20) and (21).

\subsection{Comparison between the exact and the approximate solutions}

In this section, the exact solution given by eqs (31)-(34) is compared with the approximate solution of eqs (42)-(44) in order to evaluate the ability of the approximate solution to model the SH response of the vadose zone. Figs 10 and 11 show time traces of the electric and magnetic fields, respectively, registered at a receiver located $10 \mathrm{~m}$ below the surface, computed using the exact solution and its approximation. The difference between both fields is also plotted with an enlarged scale. In this case, the plot corresponds to the total field (note that, as was pointed out before, the amplitudes of E-Cos and H-IR of the water table in the unsaturated zone are almost negligible compared to the other events). As we mention in the 'Approximate solution' section, for depths up to $\simeq 100 \mathrm{~m}$, the approximations made work fairly good. As a result, the traces computed for both solutions at a depth of $10 \mathrm{~m}$ give a very good match, and the difference between the solution and its approximation is lower than $\simeq 4$ per cent of the field in both cases. Note in Fig. 11 that the event located at $\simeq 0.09 \mathrm{~s}$ corresponds to the 

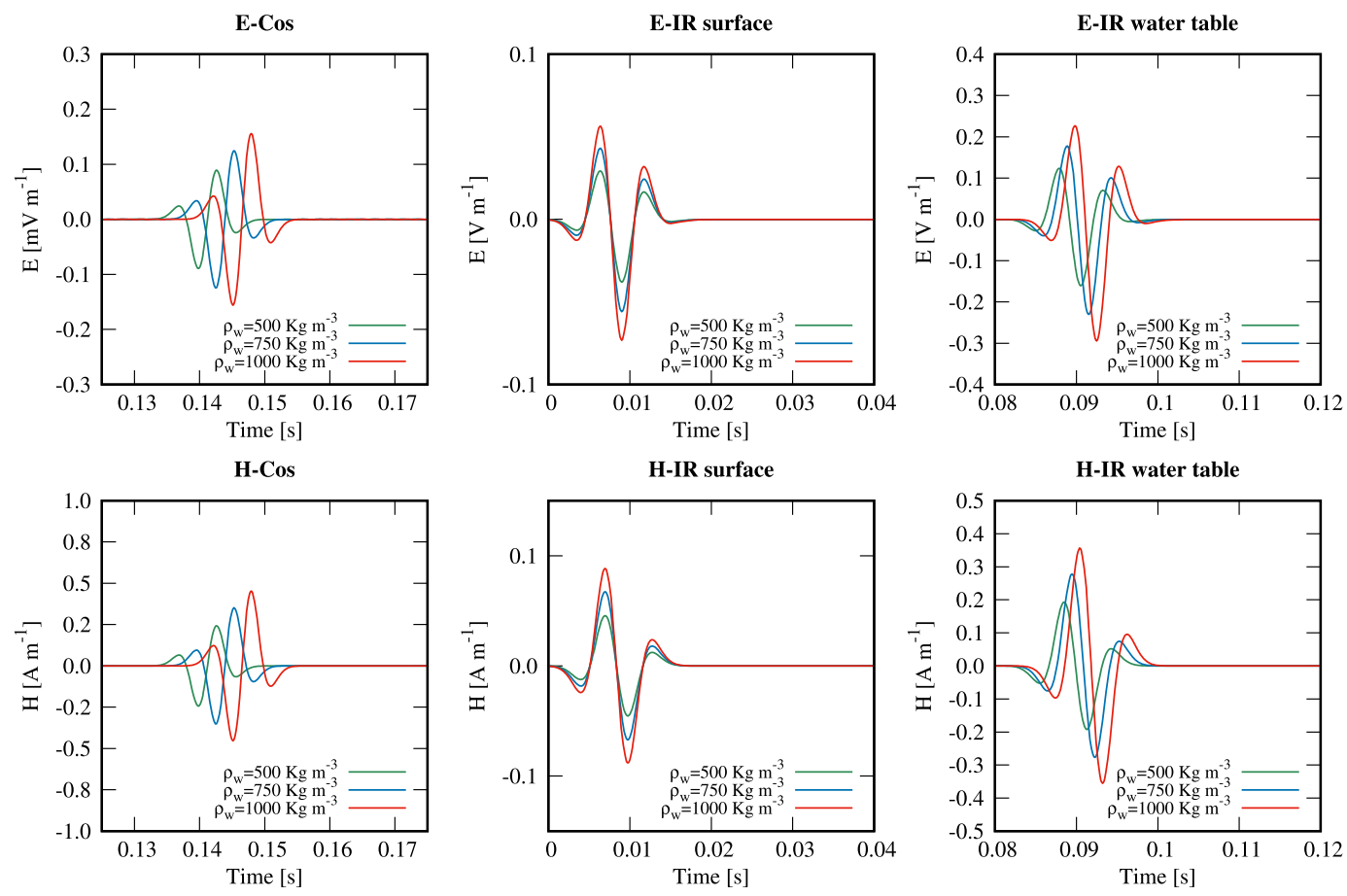

Figure 9. Time variation of the coseismic (left), interfacial response of the surface (center) and interfacial response of the water table (right) of both electric (top) and magnetic (bottom) fields registered at $z=40 \mathrm{~m}$ ( $15 \mathrm{~m}$ below the water table) for three different density values considered in this study.

\section{E field at $\mathrm{z}=10 \mathrm{~m}$}

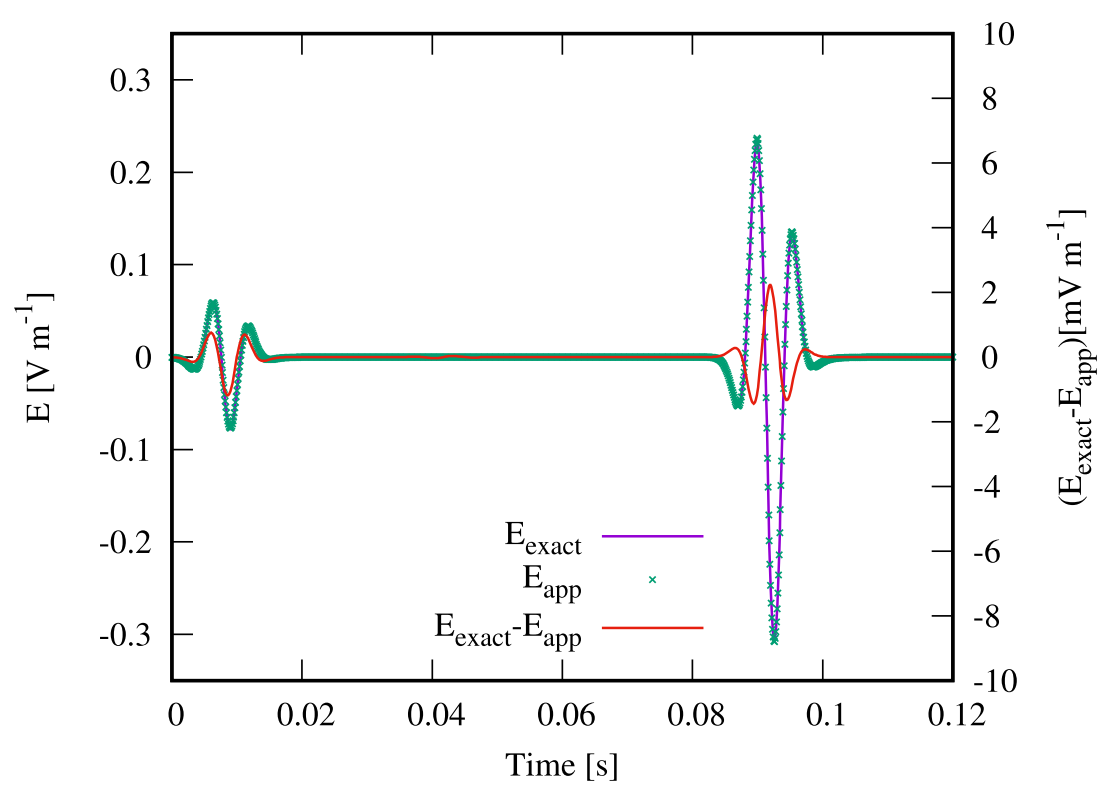

Figure 10. Comparison of the exact $\left(E_{\text {exact }}\right)$ and approximate $\left(E_{\text {app }}\right)$ solutions for the electric field response registered at $z=10 \mathrm{~m}$ below the surface. Note that a double scale is used; the left one to plot both analytical solutions and the right one to plot the difference between both solutions.

difference between the IR of the water table predicted by the exact solution and the one predicted by the approximate solution. The amplitude of this difference is $\simeq 1 \mathrm{~mA} \mathrm{~m}^{-1}$, which is almost 100 times lower than the exact solution.

The time traces of the electric and magnetic fields registered at a receiver located $100 \mathrm{~m}$ below the surface are shown in Figs 12 and 13. Note that for this depth, the coseismic components are no longer present within the considered time window. The coseismic component of the $H$ field is present in Fig. 11, but the one corresponding to the $E$ field is not visible in Fig. 10 because of its very small amplitude in comparison with the E-IR. As was expected, in the case of $z=100 \mathrm{~m}$ there is a discrepancy between both solutions, and their difference is around 25 per cent of the field. However, even for this rather big value of $z$, the approximate solution works fairly good. This example shows that the approximate solution is a very good one and in most cases, with the only restrictions imposed by the assumptions of the model, can be safely used to model the $S H$ response of the vadose zone . 


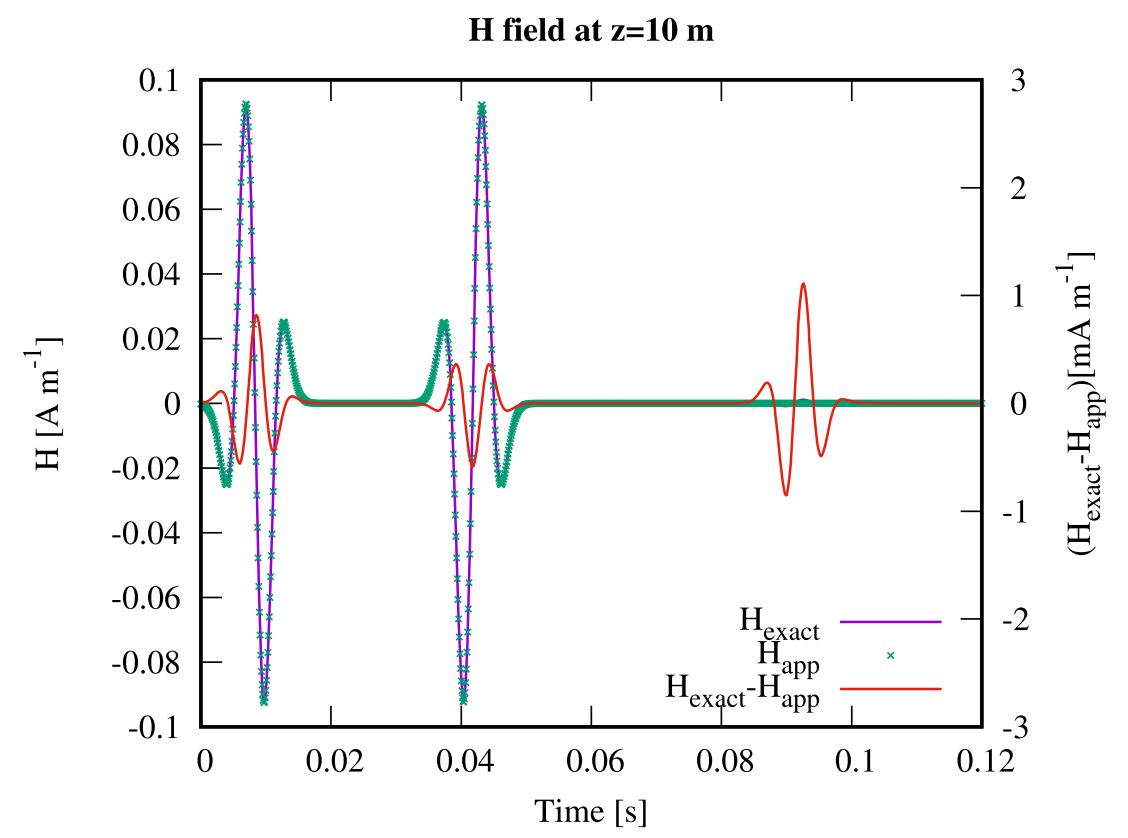

Figure 11. Comparison of the exact $\left(H_{\text {exact }}\right)$ and approximate $\left(H_{\text {app }}\right)$ solutions for the magnetic field response registered at $z=10 \mathrm{~m}$ below the surface. Note that a double scale is used; the left one to plot both analytical solutions and the right one to plot the difference between both solutions. The event located at $\simeq$ $0.09 \mathrm{~s}$ corresponds to the IR of the water table. Note that the amplitude is $\simeq 1 \mathrm{~mA} \mathrm{~m}{ }^{-1}$, which is almost 100 times lower than the exact solution.

E field at $\mathrm{z}=\mathbf{1 0 0} \mathrm{m}$

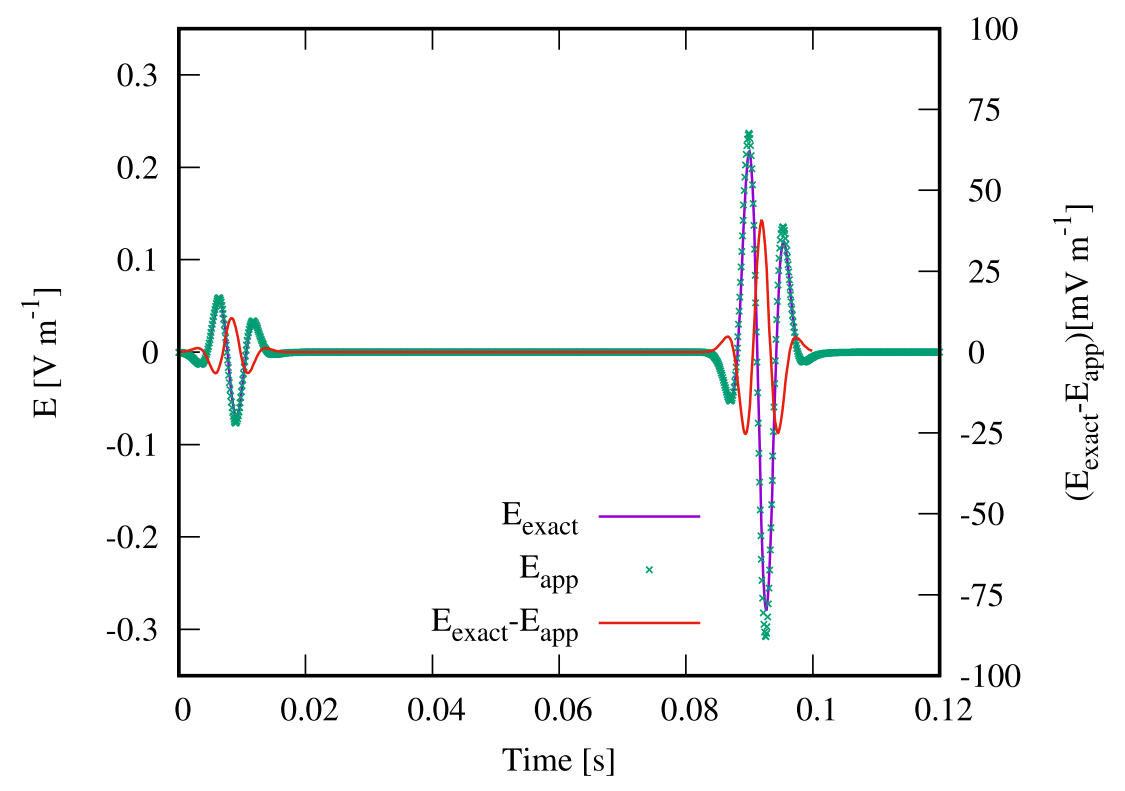

Figure 12. Comparison of the exact $\left(E_{\text {exact }}\right)$ and approximate $\left(E_{\text {app }}\right)$ solutions for the electric field response registered at $z=100 \mathrm{~m}$ below the surface. Note that a double scale is used; the left one to plot both analytical solutions and the right one to plot the difference between both solutions.

\subsection{Analysis of the approximate solution}

Finally, taking advantage of the simplicity of the analytical expressions of the approximate solution, we perform an analysis of the dependence of the electromagnetic response in terms of the physical parameters of the medium. First, let us take a look at eq. (42) which gives the approximate expression of the electric field. Note that as a consequence of the approximations made in Section 3.3, the coseismic response is absent. The reason is that, as we showed before, this component of the field is several orders of magnitude lower than the interface response. As a result, the approximated electric field is a pure IR field. The first term involving $J_{0}$ corresponds to the IR of the surface, meanwhile the second and third terms constitute the water table IR. Note that the magnitude of the IR of the surface is proportional to $J_{0}$, leading to stronger responses for higher values of $\left|J_{0}\right|$. On the other hand, the IR of the water table is proportional to the difference between and $J_{2}^{\mathrm{wt}} / \lambda_{2}$, and given that the wavenumbers $\lambda_{1}$ and $\lambda_{2}$ verify $\lambda_{1} \simeq \lambda_{2}$, this response will be mainly controlled by the difference between current densities 


\section{$\mathrm{H}$ field at $\mathrm{z}=100 \mathrm{~m}$}

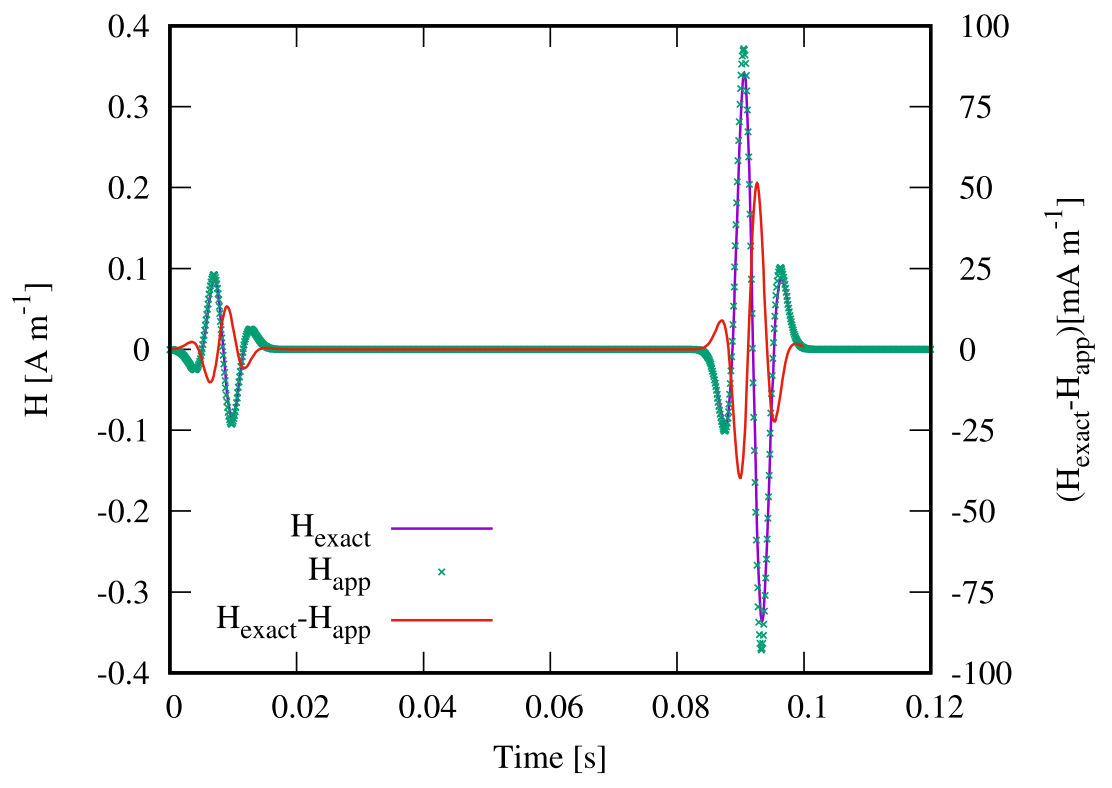

Figure 13. Comparison of the exact $\left(H_{\text {exact }}\right)$ and approximate $\left(H_{\text {app }}\right)$ solutions for the magnetic field response registered at $z=100 \mathrm{~m}$ below the surface. Note that a double scale is used; the left one to plot both analytical solutions and the right one to plot the difference between both solutions.

$J_{2}^{\mathrm{wt}}-J_{1}^{\mathrm{wt}}$. This explains the fact that for lower water saturations, the IR of the surface tends to be lower, but the one corresponding to the water table turns to be higher: $J_{0}$ is lower for lower values of $S_{\mathrm{w}}$, and the same occurs to $J_{1}^{\mathrm{wt}}$. Then, given that $J_{1}^{\mathrm{wt}}$ is lower than $J_{2}^{\mathrm{wt}}$, the difference $J_{2}^{\mathrm{wt}}-J_{1}^{\mathrm{wt}}$ will be higher for lower $S_{\mathrm{w}}$.

The approximate solution for the magnetic field over the water table is given by eq. (43). As it can be seen, this field preserves the coseismic component, given by the second term, and the interface response is purely due to the surface. As was shown before, the IR of the magnetic field is almost negligible for receivers located over the water table, and it is neglected, and therefore taken out in the approximation.

Finally, eq. (44) shows the approximate expression of the magnetic field proposed for receivers located below the water table. This field contains contributions from both the coseismic field and IRs, being the latter originated at both interfaces. Note that the IR of the water table is, as in the case of the electric field, proportional to the jump in current density $J_{2}^{\mathrm{wt}}-J_{1}^{\mathrm{wt}}$, controlling the behaviour of the magnetic field in the same way that it does for the electric field. For low values of water saturation of the vadose zone, both $J_{0}$ and $J_{1}^{\mathrm{wt}}$ will be small compared with $J_{2}^{\mathrm{wt}}$, and as a result, the amplitude of the IR of the water table will be almost the same as the amplitude of the coseismic response.

\section{CONCLUSIONS}

We found new analytical solutions - derived under the assumptions of a $S H$-wave seismic source acting on an infinite plane on the surface of the 1D Earth - allowing to straightforwardly study the seismoelectromagnetic interface and coseismic responses, and their dependence on the physical medium parameters. Moreover, we considered two layers, differing only in the fluid saturation. The upper one, of finite width, is partially saturated with a constant saturation and overlies a fully saturated semispace. An important feature of the obtained solution is that the interfacial and coseismic responses are explicitly given in the mathematical expressions of the electromagnetic fields, so they can be analysed separately. Another important feature is that the analytical solution predicts the existence of an IR originated at the surface. To our knowledge, this is the first time that its existence is analytically predicted. An approximation of the analytical solution was derived, which provides a simple way to interpret and analyse the dependence of the seismoelectric response on the various parameters of the medium. We showed that the approximate solution works fairly well in most cases of shallow exploration, given that the conditions under which the approximations work are valid for depths close to, but not higher than $100 \mathrm{~m}$, approximately. Our analysis of the dependence of the electromagnetic response on the water saturation is in complete agreement with our previous work (Zyserman et al. 2017a), showing that a sharp contrast in water saturation at the water table can induce strong interfacial responses in both fields, and in the case of the electric field, the amplitude of the interfacial response is at least three orders of magnitude higher than the coseismic response in the unsaturated zone. The sensitivity analysis has shown that the considered parameters can produce variations, both in amplitude and time shift of the registered fields. The amplitude variations can mostly be explained in terms of the variation in the magnitude of the jump in the current densities at the interfaces, meanwhile the time shifts are attributed to variations in the seismic velocity induced by the variations in the considered parameters. Although these results were obtained for a particular soil, namely a poorly consolidated silty loam, no significative change is expected in the observed qualitative behaviour of the obtained results when considering other soil textures.

Finally, it is important to mention that whether a $1 \mathrm{D}$ approach is performed, the question if the simplicity of the model yields characteristics only proper to $1 \mathrm{D}$ representation arises. One possible way to answer this question is to run 2D or 3D simulations and compare them with the 
1D predictions. However, this task is far beyond the scope of this paper, although it certainly could be the subject of forthcoming studies. Despite this, the results obtained could be very accurate in modelling the electrokinetic effects induced by a pure shear wave in the case of normal incidence. Although the cylindrical/spherical spreading experienced by the wave in its way from the source to the water table would not be modelled, the physical process for the generation of the interface response will be clearly explained by the jump in current density occurring at the water table, as it is predicted by the proposed model.

\section{ACKNOWLEDGEMENTS}

We thank the comments of two anonymous reviewers, who contributed to largely improve this work. FZ acknowledges support from CONICET through grant PIP 112-201501-00192. LBM acknowledges INSU-CNRS and the University of Strasbourg for their support.

\section{REFERENCES}

Ageeva, O.A., Svetov, B.S., Sherman, G.K. \& Shipulin, V., 1999. E-effect in rocks, Russian Geol. Geophys., 64, 1349-1356.

Allègre, V., Jouniaux, L., Lehmann, F. \& Sailhac, P., 2010. Streaming Potential dependence on water-content in fontainebleau sand, Geophys. $J$. Int., 182, 1248-1266.

Allègre, V., Lehmann, F., Ackerer, P., Jouniaux, L. \& Sailhac, P., 2012. Modelling the streaming potential dependence on water content during drainage: 1. A 1D modelling of SP using finite element method, Geophys. J. Int., 189, 285-295.

Allègre, V., Maineult, A., Lehmann, F., Lopes, F. \& Zamora, M., 2014. Selfpotential response to drainage-imbibition cycles, Geophys. J. Int., 197, $1410-1424$.

Allègre, V., Jouniaux, L., Lehmann, F., Sailhac, P. \& Toussaint, R., 2015. Influence of water pressure dynamics and fluid flow on the streamingpotential response for unsaturated conditions, Geophys. Prospect., 63, 694-712.

Bear, J., 1988. Dynamics of Fluids in Porous Media, Dover.

Beilecke, T., Krawczyk, C., Ziesch, J. \& Tanner, D., 2016. Near-surface fault detection using high-resolution shear wave reflection seismics at the CO2CRC Otway Project site, Australia, J. geophys. Res.: Solid Earth, 121(9), 6510-6532.

Biot, M.A., 1956. Theory of propagation of elastic waves in a fluid-saturated porous solid: I. low frequency range, J. acoust. Soc. Am., 28(2), 168-178.

Bordes, C., Sénéchal, P., Barrière, J., Brito, D., Normandin, E. \& Jougnot, D., 2015. Impact of water saturation on seismoelectric transfer functions: a laboratory study of coseismic phenomenon, Geophys. J. Int., 200, 13171335.

Brovelli, A., Cassiani, G., Dalla, E., Bergamini, F., Pitea, D. \& Binley, A.M., 2005. Electrical properties of partially saturated sandstones: novel computational approach with hydrogeophysical applications, Wat. Resour. Res.., 41, doi:10.1029/2004WR003628.

Carcione, J., Seriani, G. \& Gei, D., 2003. Acoustic and electromagnetic properties of soil saturated with salt water and NAPL, J. appl. Geophys., 52, 177-191.

Carsel, R. \& Parrish, R., 1998. Developing joint probability distributions of soil water characteristics, Wat. Resour. Res., 24, 755-769.

Comina, C., Krawczyk, C., Polom, U. \& Socco, L., 2017. Integration of SH seismic reflection and Love-wave dispersion data for shear wave velocity determination over quick clays, Geophys. J. Int., 210(3), 1922-1931.

Dupuis, J.C., Butler, K.E. \& Kepic, A.W., 2007. Seismoelectric imaging of the vadose zone of a sand aquifer, Geophysics, 72, A81-A85.

Dupuis, J.C., Butler, K.E., Kepic, A.W. \& Harris, B.D., 2009. Anatomy of a seismoelectric conversion: measurements and conceptual modeling in boreholes penetrating a sandy aquifer, J. Geophys. Res. Solid Earth, 114(B13), B10306.

Dupuy, B., Garambois, S. \& Virieux, J., 2016. Estimation of rock physics properties from seismic attributes - Part 1: strategy and sensitivity analysis, Geophysics, 80(3), M35-M53.

Fiorentino, E., Toussaint, R. \& Jouniaux, L., 2017. Two-phase lattice boltzmann modelling of streaming potentials: influence of the gas-water interface on the electrokinetic coupling, Geophys. J. Int., 208, 1139-1156.

Frenkel, J., 1944. On the theory of seismic and electroseismic phenomena in a moist soil, J. Phys., 8(4), 230-241.
Gao, Y. \& Hu, H., 2010. Seismoelectromagnetic waves radiated by a double couple source in a saturated porous medium, Geophys. J. Int., 181, 873-896.

Gao, Y., Huang, F. \& Hu, H., 2017. Comparison of full and quasi-static seismoelectric analytically-based modeling, J. geophys. Res.: Solid Earth, 122 (10), 8066-8106.

Garambois, S. \& Dietrich, M., 2001. Seismoelectric wave conversions in porous media: field measurements and transfer function analysis, Geophysics, 66, 1417-1430.

Gaskarov, I. \& Parkhomenko, E., 1974. The seismoelectric effect in rocks and the preconditions for its application in geological prospecting work, Izv. Akad. Sci. USSR, Physics Solid Earth, 1, 71-74.

Guan, W., Hu, H. \& Wang, Z., 2013. Permeability inversion from lowfrequency seismoelectric logs in fluid-saturated porous formations, Geophys. Prospect., 61, 120-133.

Haartsen, M.W. \& Pride, S., 1997. Electroseismic waves from point sources in layered media, J. geophys. Res., 102, 24,745-24,769.

Haines, S.H. \& Pride, S.R., 2006. Seismoelectric numerical modeling on a grid., Geophysics, 71(6), 57-65.

Haines, S.S., Pride, S.R., Klemperer, S.L. \& Biondi, B., 2007. Seismoelectric imaging of shallow targets, Geophysics, 72, G9-G20.

Holzhauer, J., Brito, D., Bordes, C., Brun, Y. \& Guatarbes, B., 2016. Experimental quantification of the seismoelectric transfer functions and its dependence on conductivity and saturation in loose sand, Geophys. Prospect., 65 (4), doi.org/10.1111/1365-2478.12448

Hornbostel, S. \& Thompson, A., 2007. Waveform design for electroseismic exploration, Geophysics, 72(2), Q1-Q10.

Hu, H. \& Gao, Y., 2011. Electromagnetic field generated by a finite fault due to electrokinetic effect, J. geophys. Res., 116, 1132-1143.

Hu, H. \& Liu, J., 2002. Simulation of the converted electric field during acoustoelectric logging, 72nd SEG Technical Program Expanded Abstracts 2002, Salt Lake City, UT, pp. 348-351.

Hu, H., Guan, W. \& Harris, J., 2007. Theoretical simulation of electroacoustic borehole logging in a fluid-saturated porous formation, J. acoust. Soc. Am., 122, 135-145.

Ivanov, A., 1939. Effect of electrization of earth layers by elastic waves passing through them, Doklady Akademii Nauk SSR (in Russian), 24, 42-45.

Jardani, A. \& Revil, A., 2015. Seismoelectric couplings in a poroelastic material containing two immiscible fluid phases, Geophys. J. Int., 202(2), $850-870$.

Johnson, D.L., Koplik, J. \& Dashen, R., 1987. Theory of dynamic permeability in fluid saturated porous media, J. Fluid. Mech., 176, 379-402.

Jouniaux, L. \& Ishido, T., 2012. Electrokinetics in earth sciences: a tutorial, Int. J. Geophysics, 2012(2012), 286107.

Jouniaux, L. \& Zyserman, F., 2016. A review on electrokinetically induced seismo-electrics, electro-seismics, and seismo-magnetics for Earth sciences, Solid Earth, 7, 249-284.

Kepic, A. \& Rosid, M., 2004. Enhancing the seismoelectric method via a virtual shot gather, 74th SEG Technical Program Expanded Abstracts, Denver, pp. 1337-1340.

Konstantaki, L., Draganov, D., Heimovaara, T. \& Ghose, R., 2013. Imaging scatterers in landfills using seismic interferometry, Geophysics, $\mathbf{7 8}(6)$, EN107-EN116. 
Konstantaki, L., Ghose, R., Draganov, D., Diaferia, G. \& Heimovaara, T., 2015. Characterization of a heterogeneous landfill using seismic and electrical resistivity data, Geophysics, 80(1), EN13-EN25.

Krawczyk, C., Polom, U., Trabs, S. \& Dahm, T., 2012. Sinkholes in the city of Hamburg-New urban shear-wave reflection seismic system enables high-resolution imaging of subrosion structures, J. appl. Geophys., 78, 133-143.

Krawczyk, C., Polom, U. \& Beilecke, T., 2013. Shear-wave reflection seismics as a valuable tool for near-surface urban applications, Leading Edge, 32(3), 253-263.

Kümpel, H., 1991. Poroelasticity: parameters reviewed, Geophys. J. Int., 105, 783-799.

Liu, X. \& Greenhalgh, S., 2014. Reflection and transmission coefficients for an incident plane shear wave at an interface separating two dissimilar poroelastic solids, Pure appl. Geophys., 171, 2111-2127.

Long, L.T. \& Rivers, W.K., 1975. Field measurement of the electroseismic response, Geophysics, 40, 233-245.

Mavko, G., Mukerji, T. \& Dvorkin, J., 2009. The Rock Physics Handbook: Tools for Seismic Analysis of Porous Media, Cambridge University Press.

Mochizuki, S., 1982. Attenuation in partially saturated rocks, J. geophys. Res., 87, 8598-8604.

Munch, F. \& Zyserman, F., 2016. Detection of non-aqueous phase liquids contamination by SH-TE seismoelectrics: a computational feasibility study, J. appl. Geophys., 130, 8-22.

Parkhomenko, E. \& Gaskarov, I., 1971. Borehole and laboratory studies of the seismoelectric effect of the second kind in rocks, Izv. Akad. Sci. USSR, Physics Solid Earth, 9, 663-666.

Parkhomenko, I. \& Tsze-San, C., 1964. A study of the influence of moisture on the magnitude of the seismoelectric effect in sedimentary rocks by a laboratory method, Bull. (Izv.) Acad. Sci., USSR, Geophys. Ser., 115-118.

Peng, R., Di, B., Wei, J., Ding, P., Zhao, J., Pan, X. \& Liu, Z., 2017. Experimental study of the seismoelectric interface response in wedge and cavity models, Geophys. J. Int.

Perrier, F. \& Morat, P., 2000. Characterization of electrical daily variations induced by capillary flow in the non-saturated zone, Pure appl. Geophys., 157, 785-810.

Pride, S., 1994. Governing equations for the coupled electromagnetics and acoustics of porous media, Phys. Rev. B: Condens. Matter, 50, 1567815695.

Pride, S. \& Garambois, S., 2005. Electroseismic wave theory of Frenkel and more recent developments, J. Eng. Mech., 131(9), 697-706.

Pride, S. \& Haartsen, M.W., 1996. Electroseismic wave properties, J. acoust. Soc. Am., 100, 1301-1315.

Pride, S. \& Morgan, F.D., 1991. Electrokinetic dissipation induced by seismic waves, Geophysics, 56(7), 914-925.

Pride, S.R., 2005. Chapter 9: Relationships between seismic and hydrological properties, in Hydrogeophysics, pp. 253-291, ,eds Yubin, Y. \& Hubbard, S., Springer.

Pugin, A.J., Pullan, S.E. \& Hunter, J.A., 2013. Shear-wave high-resolution seismic reflection in Ottawa and Quebec City, Canada, TLE, 32(3), 250255.

Revil, A., Naudet, V., Nouzaret, J. \& Pessel, M., 2003. Principles of electrography applied to self-potential electrokinetic sources and hydrogeological applications, Wat. Resour. Res., 36, 3-1-3-15.

Revil, A., Linde, N., Cerepi, A., Jougnot, D., Matthäi, S. \& Finsterle, S., 2007. Electrokinetic coupling in unsaturated porous media, J. Colloid Interface Sci., 313, 315-327.

Santos, J., Ravazzoli, C., Gauzellino, P., Carcione, J. \& Cavallini, F., 2004. Simulation of waves in poro-viscoelastic rocks saturated by immiscible fluids. Numerical evidence of a second slow wave., J. Comput. Acoust.,
$12,1-21$.

Santos, J., Ravazzoli, C., Gauzellino, P. \& Carcione, J., 2005. Numerical simulation of ultrasonic waves in reservoir rocks with patchy saturation and fractal petrophysicas properties, Comput. Geosci., 9, 1-27.

Schakel, M., Smeulders, D., Slob, E. \& Heller, H., 2012. Seismoelectric fluid/porous-medium interface response model and measurements, Transport Porous Media, 93, 271-282.

Smeulders, D.M.J., Grobbe, N., Heller, H.K.J. \& Schakel, M., 2013. Seismoelectric conversion for the detection of porous medium interfaces between wetting and nonwetting fluids, Vadose Zone J.

Strahser, M., Jouniaux, L., Sailhac, P., Matthey, P.-D. \& Zillmer, M., 2011. Dependence of seismoelectric amplitudes on water-content, Geophys. J. Int., 187, 1378-1392.

Strahser, M. H.P., Rabbel, W. \& Schildknecht, F., 2007. Polarisation and slowness of seismoelectric signals: a case study, Near Surface Geophys., 5, 97-114.

Stucchi, E., Tognarelli, A. \& Ribolini, A., 2017. SH-wave seismic reflection at a landslide (Patigno, NW Italy) integrated with $P$-wave, $J$. appl. Geophys., 146(Supplement C), 188-197.

Teja, A.S. \& Rice, P., 1981. Generalized corresponding states method for the viscosities of liquid mixtures, Ind. Eng. Chem. Fund., 20(1), 77-81.

Thompson, A. et al., 2005. Field tests of electroseismic hydrocarbon detection, SEG Technical Program Expanded Abstracts, pp. 565-568.

Thompson, A., Sumner, J. \& Hornbostel, S., 2007. Electromagnetic-toseismic conversion: a new direct hydrocarbon indicator, Leading Edge, 26, 428-435.

Thompson, A.H. \& Gist, G.A., 1993. Geophysical applications of electrokinetic conversion, Leading Edge, 12, 1169-1173.

United States Department of Agriculture, 1987, Soil Mechanics Level 1, Module 3, USDA Soil Textural Classification Study Guide. USDA Soil Conservation Service, Washington DC, United States Department of Agriculture.

Valuri, J., Dean, T. \& Dupuis, J., 2012. Seismoelectric acquisition in an arid environment, 22nd International Geophysical Conference and Exhibition, Brisbane, Australia.

Warden, S., Garambois, S., Sailhac, P., Jouniaux, L. \& Bano, M., 2012. Curvelet-based seismoelectric data processing, Geophys. J. Int., 190, $1533-1550$.

Warden, S., Garambois, S., Jouniaux, L., Brito, D., Sailhac, P. \& Bordes, C., 2013. Seismoelectric wave propagation numerical modeling in partially saturated materials, Geophys. J. Int., 194, 1498-1513.

Zimmer, M.A., Prasad, M., Mavko, G. \& Nur, A., 2007. Seismic velocities of unconsolidated sands: Part 2 - influence of sorting- and compactioninduced porosity variation, Geophysics, 72, E15-E25.

Zyserman, F., Gauzellino, P. \& Santos, J., 2010. Finite element modeling of SHTE and PSVTM electroseismics, J. appl. Geophys., 72, 79-91.

Zyserman, F., Gauzellino, P. \& Santos, J., 2012. Numerical evidence of gas hydrate detection by means of electroseismics, J. appl. Geophys., 86, 98-108.

Zyserman, F., Jouniaux, L., Warden, S. \& Garambois, S., 2015. Borehole seismoelectric logging using a shear-wave source: possible application to $\mathrm{CO}_{2}$ disposal? Int. J. Greenhouse Gas Control, 33, 82-102.

Zyserman, F., Monachesi, L. \& Jouniaux, L., 2017a. Dependence of shear wave seismoelectrics on soil textures: a numerical study in the vadose zone, Geophys. J. Int., 208(2), 918-935.

Zyserman, F., Monachesi, L. \& Jouniaux, L., 2017b. Reply to 'Comment on "Dependence of shear wave seismoelectrics on soil textures: a numerical study in the vadose zone by F.I. Zyserman, L.B. Monachesi and L. Jouniaux" by Revil, A.', Geophys. J. Int., 210 (3), 1652-1658.

\section{APPENDIX A}

From eq. (14), it is possible to obtain the following relation between $u_{s}$ and $u_{f}$ :

$u_{f}=-\frac{\omega^{2} \rho_{f}}{\left[i \omega \frac{\eta}{\kappa}-\omega^{2} g_{0}\right]} u_{s}$. 
Replacing eq. (A1) in eq. (13), one obtains:

$\frac{d^{2} u_{s}}{d z^{2}}+\lambda^{2} u_{s}=-\frac{F^{s}}{G} \delta(z)$

where $\lambda$ is given by eq. (16). Using Laplace Transform, it is possible to show that the solution for $u_{s}(z)$ is given by:

$u_{s}(z)=\left[-\frac{F^{s}}{\lambda G} \sin (\lambda z)+u_{s}(0) \cos (\lambda z)+\frac{u_{s}^{\prime}(0)}{\lambda} \sin (\lambda z)\right] \mathcal{H}(z)$,

being $\mathcal{H}(z)$ the Heaviside function. Writing the sine and cosine functions in their exponential form and regrouping terms in eq. (A3), we obtain:

$u_{x, 1}(z)=\left[-\frac{F^{s}}{2 i \lambda G}+\frac{u_{s}(0)}{2}+\frac{u_{s}^{\prime}(0)}{2 i \lambda_{1}}\right] e^{i \lambda z} \mathcal{H}(z)+\left[\frac{F^{s}}{2 i \lambda G}+\frac{u_{s}(0)}{2}-\frac{u_{s}^{\prime}(0)}{2 i \lambda_{1}}\right] e^{-i \lambda z} \mathcal{H}(z)$,

Choosing the square root of $\lambda^{2}$ that verifies $\operatorname{Re}(\lambda)<0$, then, the first term of the last equation represents a wave that travels downwards, meanwhile the second one is a wave travelling upwards. If we consider only waves travelling downwards, then the second term of eq. (A4) should vanish:

$\left[\frac{F^{s}}{2 i \lambda G}+\frac{u_{s}(0)}{2}-\frac{u_{s}^{\prime}(0)}{2 i \lambda}\right]=0$

Using the last expression in eq. (A4), we obtain:

$u_{s}(z)=u_{s}(0) e^{i \lambda z} \mathcal{H}(z)$.

Now, it remains to determine the value of the constant $u_{s}(0)$. If we assume that the solid matrix is in perfect contact with the source generator at the surface, then the shear stress applied by the source over the surface $F^{s}$ should be equal to $\tau_{x z}=-2 G_{1} \epsilon_{x z}=-G_{1} \frac{\partial u_{x, 1}}{\partial z}$ (the minus sign indicates that the shear stress is computed taking the normal in the $-z$-direction). Then, taking the first derivative of $u_{s}(z)$ with respect to $z$ in eq. (A6) and evaluating it in $z=0$ we can write:

$u_{s}^{\prime}(0)=i \lambda u_{s}(0)=-\frac{F_{s}}{G}$,

which leads to the final solution for $u_{s}(z)$ :

$u_{s}(z)=U_{s} e^{i \lambda z}=-\frac{F^{s}}{i \lambda G} e^{i \lambda z}, \quad z \geq 0$

A similar expression can be obtained for $u_{f}(z)$ replacing eq. (A8) in eq. (A1):

$u_{f}(z)=U_{f} e^{i \lambda z}=-\frac{\omega^{2} \rho_{f}}{\left[i \omega \frac{\eta}{\kappa}-\omega^{2} g_{0}\right]} U_{s} e^{i \lambda z}, \quad z \geq 0$.

Eqs (A8) and (A9) show that the solid and relative fluid displacements are produced by a shear wave originated at the source position travelling downwards with phase velocity given by $\omega /|\operatorname{Re}(\lambda)|$.

\section{A P PENDIX B}

For $z<0$ (over the surface) we assume that the medium is constituted by air, which is treated as a perfect insulator. Then, both the electric conductivity $\sigma$ and the electric current density will be zero and Maxwell's equations (11) and (12) reduce to:

$$
\begin{aligned}
& \frac{\partial H}{\partial z}=0, \\
& \frac{\partial E}{\partial z}+i \omega \mu_{0} H=0 .
\end{aligned}
$$

From eq. (B1), we know that $H$ is constant. Then, from eq. (B2). $E(z)=a z+b$, where $a$ and $b$ are complex constants. However, $a$ must be equal to zero in order to avoid the divergence of the electric field when $z \rightarrow-$. Then, $E$ is also a constant field, and replacing it in eq. (B2) we obtain that $H=0$ for $z<0$.

\section{A P P EN DIX C}

In this appendix, we show that the magnetic field should be continuous at the surface and at the water table. Let us assume a closed rectangular loop crossing the horizontal plane at $z=z_{\mathrm{wt}}$, as is depicted in Fig. C1. According to Ampere's law

$\oint_{\partial A} \mathbf{H} \cdot \mathbf{d l}=\iint_{A} \mathbf{j}_{\mathbf{v}} \cdot \mathbf{d A}$. 


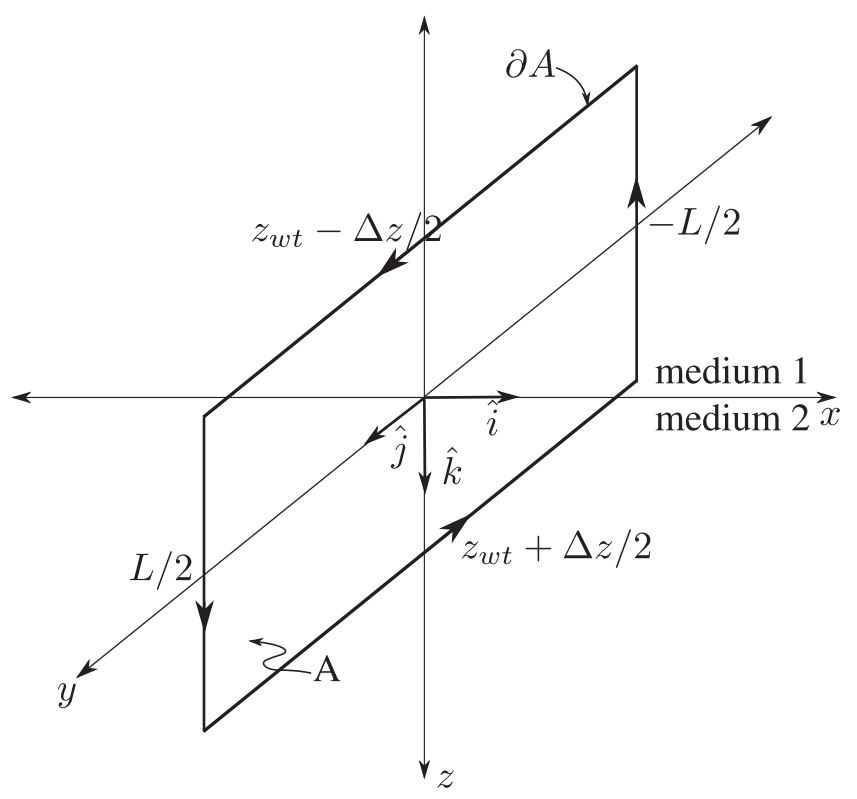

Figure C1. Continuity of $H$ across the water table $\left(z=z_{w t}\right)$ - Ampere's law.

For our model $\mathbf{H}=H(z) \hat{j}, \mathbf{j}_{\mathbf{v}}=j_{v}(z) \hat{i}$ and $\mathbf{d A}=d A \hat{i}$, so we can write:

$\int_{-L / 2}^{L / 2} H\left(z_{\mathrm{wt}}-\Delta z / 2\right) d y+\int_{L / 2}^{-L / 2} H\left(z_{\mathrm{wt}}+\Delta z / 2\right) d y=\int_{-L / 2}^{L / 2} \int_{z_{\mathrm{wt}}-\Delta z / 2}^{z_{\mathrm{wt}}+\Delta z / 2} j_{v}(z) d z d y$.

Integration over $y$ yields

$H\left(z_{\mathrm{wt}}-\Delta z / 2\right)-H\left(z_{\mathrm{wt}}+\Delta z / 2\right)=\int_{z_{\mathrm{wt}}-\Delta z / 2}^{z_{\mathrm{w} t}+\Delta z / 2} j_{v}(z) d z$.

Using the expressions for $j_{v}(z)$ given by eqs (20) and (21) we have

$H\left(z_{\mathrm{wt}}-\Delta z / 2\right)-H\left(z_{\mathrm{wt}}+\Delta z / 2\right)=\int_{z_{\mathrm{wt}}-\Delta z / 2}^{z_{\mathrm{wt}}} J_{1}^{\mathrm{wt}} e^{i \lambda_{1}\left(z-z_{\mathrm{wt}}\right)} d z+\int_{z_{\mathrm{wt}}}^{z_{\mathrm{wt}}+\Delta z / 2} J_{2}^{\mathrm{wt}} e^{i \lambda_{2}\left(z-z_{\mathrm{wt}}\right)} d z$,

which gives, after integrating over $z$

$H\left(z_{\mathrm{wt}}-\Delta z / 2\right)-H\left(z_{\mathrm{wt}}+\Delta z / 2\right)=\frac{J_{1}^{\mathrm{wt}}}{i \lambda_{1}}\left[1-e^{-i \lambda_{1} \Delta z / 2}\right]+\frac{J_{2}^{\mathrm{wt}}}{i \lambda_{2}}\left[e^{-i \lambda_{2} \Delta z / 2}-1\right]$.

Taking the limit when $\Delta z \rightarrow 0$, the right hand side of eq. (C5) tends to 0 , leading to the following relation:

$\lim _{z \rightarrow z_{\mathrm{wt}}^{-}} H(z)=\lim _{z \rightarrow z_{\mathrm{wt}}^{+}} H(z)$,

which means that the magnetic field $H$ is continuous across the water table. The same reasoning can led to the following relation valid for the continuity of the magnetic field at the surface

$\lim _{z \rightarrow 0^{-}} H(z)=\lim _{z \rightarrow 0^{+}} H(z)$.

On the other hand, given that the electric field $E(z)$ is purely tangential to both the surface and the water table interfaces, and the tangential components of the electric field should be continuous across any interface, the same will be valid for the electric field. 


\section{Key words}

Authors are requested to choose key words from the list below to describe their work. The key words will be printed underneath the summary and are useful for readers and researchers. Key words should be separated by a semi-colon and listed in the order that they appear in this list. An article should contain no more than six key words.

COMPOSITION and PHYSICAL PROPERTIES Space geodetic surveys

Composition and structure of the continental crust Tides and planetary waves

Composition and structure of the core Time variable gravity

Composition and structure of the mantle Transient deformation

Composition and structure of the oceanic crust

Composition of the planets

Creep and deformation

Defects

Elasticity and anelasticity

Electrical properties

Equations of state

Fault zone rheology

Fracture and flow

Friction

High-pressure behaviour

Magnetic properties

Microstructure

Permeability and porosity

Phase transitions

Plasticity, diffusion, and creep

\section{GENERAL SUBJECTS}

Core

Gas and hydrate systems

Geomechanics

Geomorphology

Glaciology

Heat flow

Hydrogeophysics

Hydrology

Hydrothermal systems

Instrumental noise

Ionosphere/atmosphere interactions

Ionosphere/magnetosphere interactions

Mantle processes

Ocean drilling

Structure of the Earth

Thermochronology

Tsunamis

Ultra-high pressure metamorphism

Ultra-high temperature metamorphism

GEODESY and GRAVITY

Acoustic-gravity waves

Earth rotation variations

Geodetic instrumentation

Geopotential theory

Global change from geodesy

Gravity anomalies and Earth structure

Loading of the Earth

Lunar and planetary geodesy and gravity

Plate motions

Radar interferometry

Reference systems

Satellite geodesy

Satellite gravity

Sea level change

Seismic cycle

Archaeomagnetism

Electrical anisotropy

Electromagnetic theory

Environmental magnetism

Geomagnetic excursions

Geomagnetic induction

Ground penetrating radar interpretation

Magnetostratigraphy

Magnetotellurics

Palaeointensity

Palaeomagnetism

Rapid time variations

Remagnetization magnetostratigraphy

Satellite magnetics

Downhole methods

Fourier analysis

Fractals and multifractals

Image processing

Instability analysis
GEOGRAPHIC LOCATION

Africa

Antarctica

Arctic region

Asia

Atlantic Ocean

Australia

Europe

Indian Ocean

Japan

New Zealand

North America

Pacific Ocean

South America
Interferometry

Inverse theory

Joint inversion

Neural networks, fuzzy logic

Non-linear differential equations

Numerical approximations and analysis

Numerical modelling

Numerical solutions

Persistence, memory, correlations, clustering

Probabilistic forecasting

Probability distributions

Self-organization

Spatial analysis

Statistical methods

Thermobarometry

Time-series analysis

Tomography

Waveform inversion

Wavelet transform

\section{GEOMAGNETISM and ELECTROMAGNETISM PLANETS}

Biogenic magnetic minerals

Controlled source electromagnetics (CSEM)

Dynamo: theories and simulations

Electrical resistivity tomography (ERT)

Magnetic anomalies: modelling and

Magnetic fabrics and anisotropy

Magnetic field variations through time

Magnetic mineralogy and petrology

Marine electromagnetics

Marine magnetics and palaeomagnetics

Non-linear electromagnetics

Palaeomagnetic secular variation

Reversals: process, time scale,

Rock and mineral magnetism

GEOPHYSICAL METHODS
Planetary interiors

Planetary volcanism

SEISMOLOGY

Acoustic properties

Body waves

Coda waves

Computational seismology

Controlled source seismology

Crustal imaging

Earthquake dynamics

Earthquake early warning

Earthquake ground motions

Earthquake hazards

Earthquake interaction, forecasting, and prediction

Earthquake monitoring and test-ban treaty verification

Earthquake source observations

Guided waves

Induced seismicity

Interface waves

Palaeoseismology

Rheology and friction of fault zones

Rotational seismology

Seismic anisotropy

Seismic attenuation

Seismic instruments

Seismic interferometry

Seismic noise

Seismic tomography

Site effects

Statistical seismology

Surface waves and free oscillations

Theoretical seismology

Tsunami warning
Seismicity and tectonics 
Volcano seismology

Wave propagation

Wave scattering and diffraction

\section{TECTONOPHYSICS}

Backarc basin processes

Continental margins: convergent

Continental margins: divergent

Continental margins: transform

Continental neotectonics

Continental tectonics: compressional

Continental tectonics: extensional

Continental tectonics: strike-slip and transform

Cratons

Crustal structure

Diapirism

Dynamics: convection currents, and mantle plumes

Dynamics: gravity and tectonics

Dynamics: seismotectonics

Dynamics and mechanics of faulting

Dynamics of lithosphere and mantle

Folds and folding

Fractures, faults, and high strain

deformation zones

Heat generation and transport

Hotspots
Impact phenomena

Intra-plate processes

Kinematics of crustal and mantle deformation

Large igneous provinces

Lithospheric flexure

Mechanics, theory, and modelling

Microstructures

Mid-ocean ridge processes

Neotectonics

Obduction tectonics

Oceanic hotspots and intraplate volcanism

Oceanic plateaus and microcontinents

Oceanic transform and fracture zone processes

Paleoseismology

Planetary tectonics

Rheology: crust and lithosphere

Rheology: mantle

Rheology and friction of fault zones

Sedimentary basin processes

Subduction zone processes

Submarine landslides

Submarine tectonics and volcanism

Tectonics and climatic interactions

Tectonics and landscape evolution

Transform faults

Volcanic arc processes
VOLCANOLOGY

Atmospheric effects (volcano)

Calderas

Effusive volcanism

Eruption mechanisms and flow emplacement

Experimental volcanism

Explosive volcanism

Lava rheology and morphology

Magma chamber processes

Magma genesis and partial melting

Magma migration and fragmentation

Mud volcanism

Physics and chemistry of magma bodies

Physics of magma and magma bodies

Planetary volcanism

Pluton emplacement

Remote sensing of volcanoes

Subaqueous volcanism

Tephrochronology

Volcanic gases

Volcanic hazards and risks

Volcaniclastic deposits

Volcano/climate interactions

Volcano monitoring

Volcano seismology 